\title{
Penetration of a salinity front into a rotating basin: Laboratory experiments and a simple theory
}

\author{
by O. Marchal ${ }^{1,2}$, J. A. Whitehead ${ }^{1}$ and A. Jensen ${ }^{1}$
}

\begin{abstract}
Freshwater is released along a wall of a basin containing salt water and rotating anticlockwise. The freshwater source is located near the surface between the center of the cylindrical basin and a corner along the wall. Experiments are performed with different discharge rates and the same rotation rate. The freshwater initially forms a bulge near the source, and then a buoyant gravity current bends to the right and flows along the wall toward the periphery of the basin. Separation of the current at the corner is never observed. The salinity front along the wall moves persistently away from the wall with a time scale greatly exceeding the rotation period. Its movement is compared to numerical solutions of a twolayer theory, where friction in the Ekman layer straddling the layer interface is the sole ageostrophic effect. The theory shows that the depth of the interface $(h)$ satisfies a nonlinear diffusion equation. The symmetric part of the diffusion tensor causes light fluid to move down the gradient of $h$ and represents the effect of vertical friction. The associated diffusivity reaches a maximum at $h / \delta=\pi / 2$, where $\delta$ is the Ekman layer depth. The antisymmetric part of the diffusion tensor causes light fluid to move perpendicularly to $\nabla h$ and represents the effect of geostrophic motion. The associated diffusivity increases monotonically with $h / \delta$ and greatly exceeds the diffusivity of the symmetric part if $h / \delta$ is of order of one or more. Comparison of numerical solutions with experimental data supports the theory.
\end{abstract}

\section{Recollection by Jack Whitehead}

In 1979 and 1980, I was extremely fortunate to have the opportunity to experimentally test results of a theory that Professor Stern was then finishing up. A demonstration film that we playfully entitled "Rotating Bores" (Stern, Whitehead) finished off the project. We greatly enjoyed working together, and soon developed both a close friendship and a collaboration of convenience; he used the project to support travel to the laboratory and the GFD summer school at WHOI, I picked his fruitful brain for new studies of coastal currents and eddies, and the projects always involved numerous students and colleagues. This present study, in collaboration with $\mathrm{O}$. Marchal, reminds me of those days with great satisfaction. Here, we look at a laboratory current with a balance between Coriolis, pressure and viscous forces that is found in the limit of very small volume flux. That is exactly the opposite limit to the Coriolis, pressure and inertia balance for currents with large flux that Stern investigated twenty-three years ago. I miss him and regret that we cannot show these new results to him.

1. Woods Hole Oceanographic Institution, Woods Hole, Massachusetts, 02543, U.S.A.

2. Corresponding author. email: omarchal@whoi.edu 


\section{Introduction}

The flow produced by buoyant water released along the side wall of a rotating basin has long been investigated in the laboratory (e.g., Whitehead and Miller, 1979; Stern, 1980; Griffiths and Linden, 1981). The problem is relevant to a variety of geophysical phenomena, such as light water entering the ocean from a natural dam that is suddenly broken or, equivalently, from the rapid melting of continental ice (Nof, 1987). A general observation is that the buoyant fluid turns to the right (in a basin rotating anticlockwise) and forms a buoyant gravity current along the wall (hereafter referred to as simply a gravity current).

The gravity current along the wall arises from the following reason (Griffiths, 1986). When fluid is injected along a wall, the component of the buoyancy force parallel to the wall, which is proportional to the gradient of the fluid layer thickness parallel to the wall, 'causes the injected fluid to spread along the boundary.' The condition of no normal flow at the wall implies that the component of the Coriolis force parallel to the wall vanishes. 'However, fluid spreading along the wall with the wall on its left (...) experiences a Coriolis force to its right and cannot move away from the source ... On the other side of the source, motion along the wall experiences a Coriolis force toward the wall ... and a flow proceeds along the boundary' (Griffiths, 1986).

Gravity currents produced in the laboratory with rotating tables are bounded by a density front that separates the incoming light fluid from the ambient heavier fluid. The evolution of these fronts has received considerable attention (e.g., Whitehead and Miller, 1979; Stern, 1980; Griffiths and Linden, 1981; Stern et al., 1982; Griffiths and Hopfinger, 1983; Avicola and Huq, 2002; Thomas and Linden, 2007; Gregorio et al., 2011). For example, Griffiths and Linden (1981) created a gravity current from a continuous point source that was positioned along the outer vertical wall of a cylinder placed at the center of a larger cylinder. They observed that, at any point on the wall, the depth and width of the current increased with time. The flow was diverted perpendicularly to the wall and was entirely laminar. The current, however, became eventually unstable to breaking waves. Stern et al. (1982) and Griffiths and Hopfinger (1983) generated gravity currents by removing vertical barriers which initially retained a layer of freshwater that laid at one end of a long channel and floated on a much larger volume of salt water. The resulting currents displayed three-dimensional turbulence. The width of the currents varied with distance behind the nose and increased with time. Avicola and Huq (2002) released freshwater along the rim of a cylinder containing seawater. The width of the resulting current was found to increase with time, as in subsequent work (Gregorio et al., 2011). Other aspects of gravity currents in a rotating basin have been studied in the laboratory, particularly in the context of buoyant river water or 'plumes' entering the ocean (e.g., Lentz and Helfrich, 2002; Avicola and Huq, 2003a,b; Horner-Devine et al., 2006).

The dynamics of gravity currents overlying a layer of deep water have been investigated in several theoretical studies. The volume flux of these currents is predicted to be, from 
the thermal wind balance, close to $g^{\prime} h^{2} / 2 f$, where $g^{\prime}$ is the reduced gravity based on the density contrast between the resident and incoming waters, $h$ the thickness of the current, and $f$ the Coriolis parameter. This prediction is borne out by many models that rely on energy, potential vorticity, and momentum conservation contraints (e.g., Whitehead et al., 1974; Stern, 1980; Nof, 1987; Hacker and Linden, 2002). The width of the currents naturally scales with the Rossby radius $\sqrt{g^{\prime} h} / f$, although it is observed to vary with distance along the wall (e.g., Stern et al., 1982; Griffiths and Hopfinger, 1983).

Gravity currents can be affected by the vertical shear of horizontal velocity at the interface between the buoyant water and the dense water below. Friction at the interface could eventually break down the geostrophic constraint and allow the density front to move down the pressure gradient, i.e., away from the coast (e.g., Wright, 1989). The primary objective of this study is to test the prediction that interfacial friction leads to a spreading gravity current in the laboratory. Accordingly, plumes that most readily spread by interfacial friction would have a thickness approaching the order of the Ekman layer depth, $\sqrt{2 v / f}$, where $\nu$ is the kinematic viscosity. Therefore, the limit we investigate here is for currents whose volume flux has an order of magnitude comparable to $g^{\prime} \nu / f^{2}$ (previous studies generally considered greater flux).

Another mechanism of export of buoyant water away from the coast is the separation of the gravity current near a coastline irregularity. Laboratory experiments suggest that separation at a corner occurs when the radius of curvature of the corner is less than the inertial radius of the current (e.g., Whitehead and Miller, 1979; Boormans and Garrett, 1989). A relatively well-studied current along a coast is the long and narrow current flowing equatorward along northeastern North America (for reviews see, e.g., Loder et al. (1998) and Lentz (2008)). Observations indicate that the transports of volume and freshwater in the current drop by two orders of magnitude from Cape Chidley near $60^{\circ} \mathrm{N}$ to Cape Hatteras near $35^{\circ} \mathrm{N}$ (Loder et al., 1998). It has been suggested that the drop in volume and freshwater transports results from the detachment of the current near the Tail of the Grand Banks (see Fratantoni and McCartney (2010); and references therein).

In this paper, simple experiments are conducted in the laboratory and are augmented by a theoretical model. Freshwater is discharged along the vertical wall of a basin containing salt water and rotating anticlockwise. Emphasis is placed on two aspects of the resulting gravity current along the wall: (i) the widening of the current and (ii) the possibility of current separation near an irregularity along the wall. In order to observe the widening of the current over a relatively large amount of time, freshwater is released along the wall of a basin of a relatively large size. Likewise, in order to represent a coastline irregularity such as the Grand Banks, the wall along which freshwater is discharged has a corner with a right angle. The resulting basin geometry is similar to that adopted in a laboratory study of the North Atlantic Ocean (Rossby and Nilsson, 2003) and is fully described in Section 2.

This paper is organized as follows. The laboratory experiments are described in Section 2. Details about the experimental protocol are provided and the movement of the salinity front 


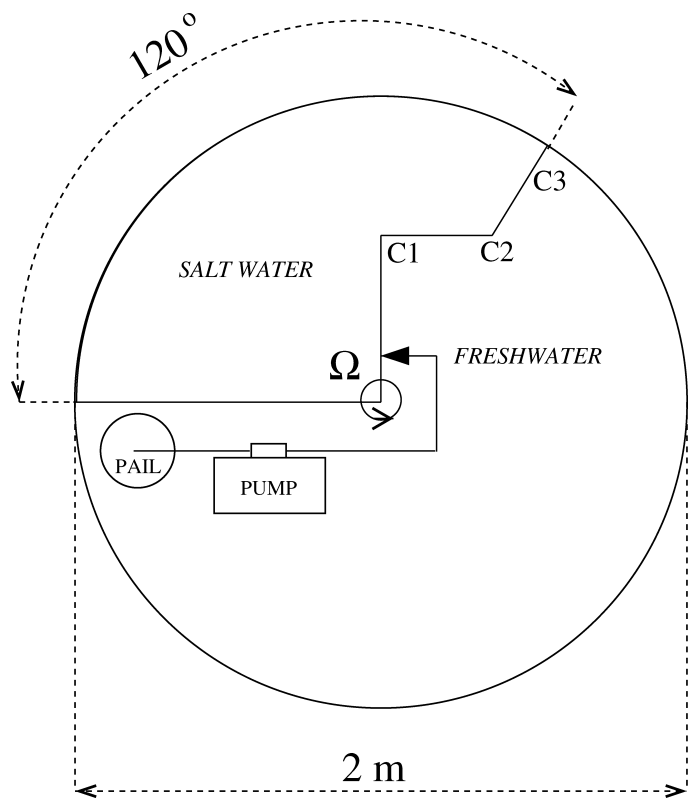

Figure 1. A top view of the rotating basin, walls, and freshwater source.

away from the wall is characterized. In Section 3 the movement of the front as observed in the laboratory is given a dynamical interpretation. A theory of a surface layer of light fluid over a deep layer of dense fluid, where the layer interface may intersect the free surface, is discussed. The theory assumes uniform rotation and includes vertical friction as the sole ageostrophic effect in both layers. Numerical solutions of the theory are obtained and compared to laboratory results in Section 4. The differences between experimental and numerical results are discussed in Section 5. A summary follows in Section 6.

\section{Laboratory experiments}

a. Protocol

A rotating table in the Geophysical Fluid Dynamics Laboratory at WHOI is used. The table supports a cylindrical tank with a radius of $1 \mathrm{~m}$ and a height of $0.45 \mathrm{~m}$. For our experiments the tank is modified to contain a sectorial basin with an angle of $120^{\circ}$ at the apex (Fig. 1). The basin has a flat white bottom and vertical walls made of foamed PVC with a height of $0.43 \mathrm{~m}$ (Fig. 2). The wall along which freshwater is released runs from the apex toward the arc of the basin. It has a corner ( $\mathrm{C} 1$ in Fig. 1) which has a right angle on the inner side and which is at a distance of $0.58 \mathrm{~m}$ from the apex. A square opening $(5 \mathrm{~cm} \times 5 \mathrm{~cm})$ is made in the wall near the apex and a sponge is embedded into the opening. Below, the wall along which freshwater is released (through the sponge) is called simply 




Figure 2. A side view of the rotating basin.

the 'wall.' The other wall joining the apex to the arc is straight and is perpendicular to the wall. It is called the 'adjacent wall.' The entire basin is covered with a polycarbonate lid.

A video camera that co-rotates with the table is used to observe the evolution of the dyed freshwater above the white floor of the basin during the experiments. The camera is mounted on top of a vertical post fixed along the outer side of the wall (corner C2 in Figs. 1 and 2). The lens of the camera is approximately facing corner $\mathrm{C} 1$ of the wall and is at a distance of about $2.11 \mathrm{~m}$ above the water surface in the basin. In this way, none of the freshwater is hidden by the walls.

The spin-up proceeds as follows. The sectorial basin is filled with salt water (seawater) to a level of about $0.23 \mathrm{~m}$ from the bottom of the basin. It is then set in anticlockwise rotation at a rate $\Omega=0.5 \mathrm{~s}^{-1}$, which corresponds to a Coriolis parameter $f=2 \Omega=1 \mathrm{~s}^{-1}$. Freshwater is pumped into the basin only after salt water in the basin is (or is close to being) at rest in the rotating frame. Then, the pump is turned on and freshwater flows into the basin through the sponge near the surface along the wall. The freshwater discharge is maintained at constant rate $q$ throughout the experiment. The experiment lasts from about 5 minutes for the largest inflow rate to about 15 minutes for the smallest inflow rate (see below). For each inflow rate, duplicate experiments are conducted in order to assess the reproducibility of the results. Further details about the laboratory set-up and spin-up are provided in Appendix A.

A density meter (DMA 58) is used to measure the densities of freshwater and salt water at $20^{\circ} \mathrm{C}$. Duplicate measurements on the same sample give a density value of $1000.22 \pm$ $0.02 \mathrm{~kg} \mathrm{~m}^{-3}$ for freshwater and $1024.09 \pm 0.07 \mathrm{~kg} \mathrm{~m}^{-3}$ for salt water (mean \pm one standard deviation). Based on these measurements, a reduced gravity $g^{\prime}=0.23 \mathrm{~m} \mathrm{~s}^{-2}$ is selected in subsequent calculations unless stipulated otherwise.

Two pumps with different ranges of flow rate are used for our experiments (Cole-Parmer and Micropump Inc.). Each pump is calibrated by measuring the volume of water delivered 
by the pump for a given reading on the pump and over a given amount of time ( 5 trials). Six different flow rates $(q)$ ranging from $1.03 \pm 0.00 \mathrm{ml} \mathrm{s}^{-1}$ to $4.51 \pm 0.08 \mathrm{ml} \mathrm{s}^{-1}$ (mean \pm one standard deviation) are considered for our experiments. The reading on the pump during our experiments is the same value as for the calibration, except for experiments with $q=$ $1.85 \pm 0.00 \mathrm{ml} \mathrm{s}^{-1}$ for which the flow rate and its standard deviation are determined by extrapolation from calibration at another reading on the same pump.

\section{b. Results}

All experiments exhibit three basic features (in chronological order): (i) a bulge that forms near the freshwater source, (ii) a buoyant current that moves along the wall toward and then along the arc of the basin, and (iii) a slow migration, away from the wall, of the salinity front between the incoming light water and the ambient dense water. To our surprise, the buoyant current does not separate from the wall at corner $\mathrm{C} 1$ in any of the experiments. Accordingly, emphasis is primarily placed on the third feature (iii). In the remainder of this Section, the movement of the salinity front is described and dimensionless numbers which characterize the buoyant current are compared with observational estimates for buoyant discharges into the coastal ocean.

We first illustrate the nature of the flow in our experiments, using as an example results from a preliminary experiment for which the salinity front was particularly regular and a color camera was used (Fig. 3). Results from this experiment are representative of those of subsequent experiments for which the front showed comparable regularity but which were recorded with a black-and-white camera. For this experiment, the rate of freshwater inflow amounts to $q=1.82 \mathrm{ml} \mathrm{s}^{-1}$ and the basin is covered with plastic instead of a polycarbonate lid. Otherwise, the experimental protocol is similar to that described in Section 2a. Figure 3 shows the spreading of buoyant water (red patch) in the rotating basin at different times after starting the freshwater release at the wall. The salinity front moves both along and away from the wall. In the context of our experiments, the internal deformation radius is defined as $l_{D}=\sqrt{g^{\prime} h_{w}} / f$, where $h_{w}$ is the depth of the buoyant layer at the wall. If the flow along the wall is in thermal wind balance with constant vertical shear, the depth of the buoyant layer at the wall is given by $h_{w}=\sqrt{2 f q / g^{\prime}}$, so $l_{D}=\left(2 g^{\prime} q / f^{3}\right)^{1 / 4}$. The parameters for the experiment displayed in Figure 3 result in $l_{D}=3.0 \mathrm{~cm}$. In comparison, the width of the gravity current at corner $\mathrm{C} 1$ is $20-24 \mathrm{~cm}$ about four minutes after the initiation of freshwater release (bottom panel of Fig. 3). This is consistent with the notion that there is considerable spreading through the frictional layer.

$i$. Movement of salinity front. All experiments are recorded on a video tape from the corotating camera. For each experiment a digitized sequence with time step of $10 \mathrm{~s}$ is produced from the video tape using the software XCAP. The sequence is used to determine the evolution of the salinity front. The distance of the front from the wall is measured in the direction normal to the wall and from corner $\mathrm{C} 1$ of the wall (see double arrow in bottom panel 



Figure 3. Distribution of buoyant water (in red) observed in the laboratory at three different times since the initiation of freshwater discharge $\left(q=1.82 \mathrm{ml} \mathrm{s}^{-1}\right)$ : ca. 50 seconds (top panel), ca. 2 minutes (middle), and ca. 4 minutes (bottom). For reference, the black and white marks on top of the wall at the bottom of each panel have a width of $2 \mathrm{~cm}$. The position of the salinity front is determined from corner $\mathrm{C} 1$ at the wall (see double arrow in bottom panel). Glittering features are produced by light reflection on the plastic surface covering the basin. 
of Fig. 3). It is determined using a graduation on top of the adjacent wall. The graduation marks are equally spaced by $4 \mathrm{~cm}$ and are located at distances ranging from $l_{\mathrm{o}}=2 \mathrm{~cm}$ to $l_{\mathrm{o}}=38 \mathrm{~cm}$ from the wall. The actual distance of the salinity front from the wall $(l)$ is obtained by correcting $l_{0}$ for a geometrical effect due the obliqueness of the line of sight between the lens of the camera and the apparent position of the front at the water surface,

$$
l=l_{\mathrm{o}} \frac{h_{\mathrm{st}}+h_{\mathrm{tl}}}{h_{\mathrm{tl}}} .
$$

Here $h_{\mathrm{st}}$ is the height of the top of the wall above the water surface and $h_{\mathrm{tl}}$ is the height of the camera lens above the top of the wall. Assuming $h_{\mathrm{st}}=0.43-0.23=0.20 \mathrm{~m}$ and $h_{\mathrm{tl}}=2.11-0.20=1.91 \mathrm{~m}$, the correction factor $l / l_{\mathrm{o}}$ is 1.10 .

The uncertainty in each value of $l$ is obtained from (1) by propagating the uncertainty in the measured distance $\left(\sigma_{l_{0}}\right)$, the uncertainty in the height of the camera lens above the water surface $\left(\sigma_{h_{\mathrm{tot}}}\right.$, where $\left.h_{\mathrm{tot}}=h_{\mathrm{st}}+h_{\mathrm{tl}}\right)$, and the uncertainty in the height of the camera lens above the top of the wall $\left(\sigma_{h_{\mathrm{t}}}\right)$, assuming no correlation between these individual uncertainties,

$$
\frac{\sigma_{l}}{l}=\sqrt{\left(\frac{\sigma_{l_{\mathrm{o}}}}{l_{\mathrm{o}}}\right)^{2}+\left(\frac{\sigma_{h_{\mathrm{tot}}}}{h_{\mathrm{tot}}}\right)^{2}+\left(\frac{\sigma_{h_{\mathrm{tl}}}}{h_{\mathrm{tl}}}\right)^{2}} .
$$

The following values are adopted: $\sigma_{l_{\mathrm{o}}}=0.5 \mathrm{~cm}$ and $\sigma_{h_{\mathrm{tot}}}=\sigma_{h_{\mathrm{tl}}}=5 \mathrm{~cm}$ (the uncertainty in $h_{\mathrm{st}}$ is taken as negligible compared to that of $\left.h_{\mathrm{tl}}\right)$. Hence the uncertainty in the actual distance of the front from the wall ranges from $\sigma_{l}=0.6 \mathrm{~cm}$ for $l_{\mathrm{o}}=2 \mathrm{~cm}$ to $\sigma_{l}=1.6 \mathrm{~cm}$ for $l_{\mathrm{o}}=38 \mathrm{~cm}$. Further details about the determination of the movement of the salinity front are reported in Appendix A.

The distance of the salinity front from the wall is displayed as a function of time for each experiment (Figs. 4a-4f). The distance is corrected for the geometrical effect described above and normalized by $\sqrt{g^{\prime} h_{0}} / f=11 \mathrm{~cm}$, where $h_{0}$ is the height of the freshwater inlet. Note that this value is larger than the deformation radius $l_{D}$, which ranges from $2.6 \mathrm{~cm}$ for the experiment with $q=1.03 \mathrm{ml} \mathrm{s}^{-1}$ to $3.8 \mathrm{~cm}$ for the experiment with $q=4.51 \mathrm{ml} \mathrm{s}^{-1}$ (Table 1). Likewise, time is normalized by the rotation period $4 \pi / f$. In all experiments, the salinity front migrates persistently away from the wall, reaching a distance from the wall of many deformation radii $l_{D}$ (Figs. $4 \mathrm{a}-4 \mathrm{f}$ ). Duplicate experiments show that results are generally reproducible for each rate of freshwater inflow in the initial stage of the experiments. In the final stage significant differences are often found. These differences arise primarily from small irregularities of the salinity front which develop as the front moves away from the wall. The precise location and amplitude of the irregularities vary from one experiment to another. One exception resides in the duplicate experiments with the lowest flow rate $\left(q=1.03 \pm 0.00 \mathrm{ml} \mathrm{s}^{-1}\right)$, where results disagree in the initial stage of the experiment and agree in the final stage. Note that the time uncertainty generally increases with distance from the wall. Indeed, the salinity front appears as more diffuse and its precise location is more difficult to determine as the front moves into the interior of the basin. 

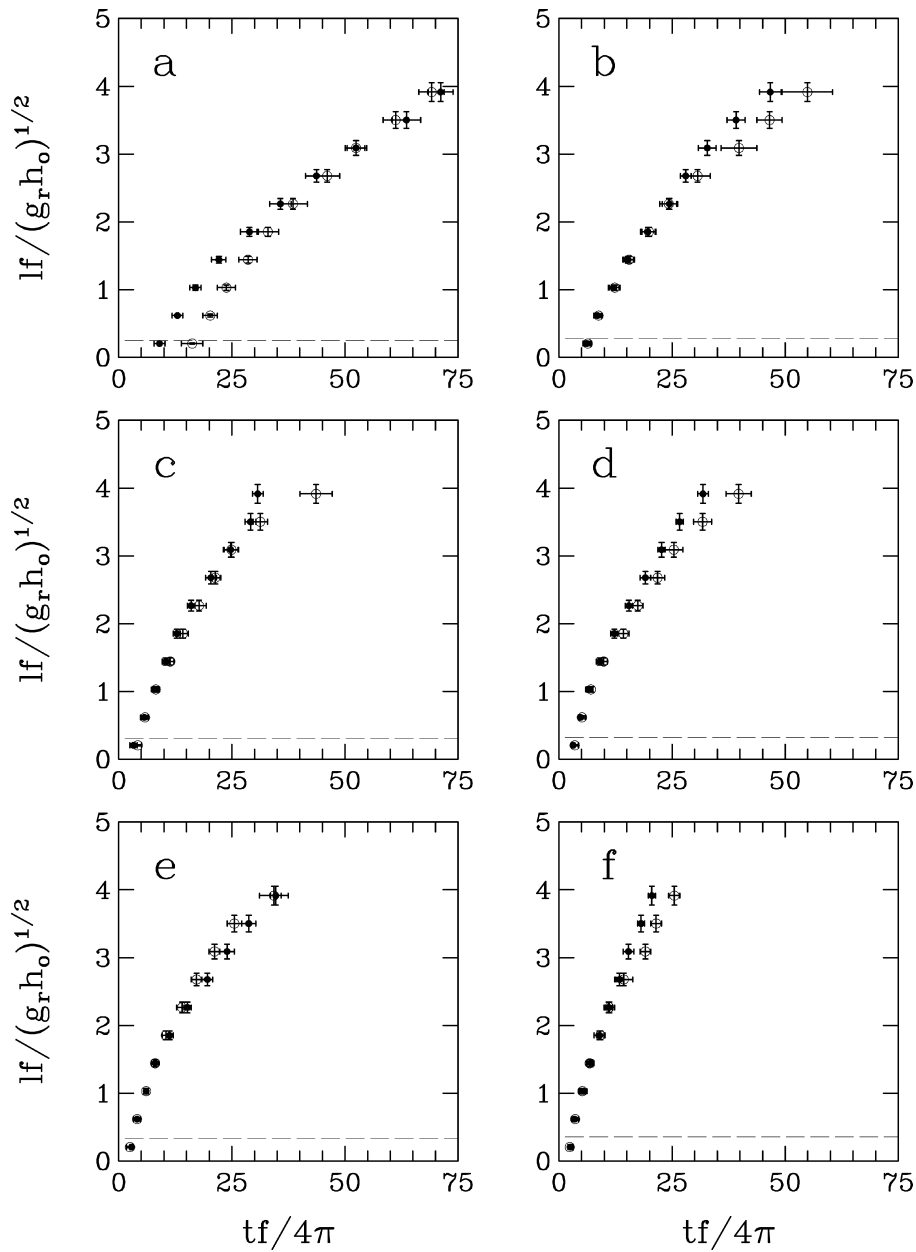

Figure 4. Experimental measurements of the distance of the salinity front from the wall $(l)$ as a function of time $(t)$ for different rates of freshwater inflow (in ml s${ }^{-1}$ ): $1.03 \pm 0.00$ (a), $1.85 \pm 0.00$ (b), $2.51 \pm 0.03$ (c), $3.05 \pm 0.02$ (d), $3.73 \pm 0.04$ (e), and $4.51 \pm 0.08$ (f). The distance from the wall (time) is normalized by $\sqrt{g^{\prime} h_{0}} / f(4 \pi / f)$, where $g^{\prime}$ is the reduced gravity ( $g_{r}$ in the figure). Results from duplicate laboratory experiments are shown by solid and open circles (horizontal and vertical bars denote uncertainty estimates). The horizontal dashed line shows the ratio $l_{D} / \sqrt{g^{\prime} h_{0}} / f$ for each experiment, where $l_{D}=\left(2 g^{\prime} q / f^{3}\right)^{1 / 4}$ is the deformation radius.

The variation with time of the distance of the salinity front from the wall can be described to a good approximation by a quadratic regression for all experiments (not shown). Such description is useful since the coefficients of the regression model $l=\alpha_{0}+\alpha_{1} t+\alpha_{2} t^{2}+\epsilon$, where $t$ is time and $\epsilon$ is an error, have an obvious kinematical interpretation. Consider first the coefficient $\alpha_{1}$, which is the speed of the front near the wall. The least-squares estimates of $\alpha_{1}$ are denoted by $a_{1}$. They are normalized by $\sqrt{g^{\prime} h_{0}}$ and displayed as a function of the 
Table 1. Laboratory experiments with flow scales and dimensionless numbers $\dagger$

\begin{tabular}{lcccccccccccc}
\hline$q^{a}$ & $l_{D}^{b}$ & $h_{w}^{c}$ & $\bar{h}^{d}$ & $K^{e}$ & $\begin{array}{c}F_{\|}^{f} \\
\times 10\end{array}$ & $\begin{array}{c}F_{\perp}^{g} \\
\times 10\end{array}$ & $\begin{array}{c}\epsilon_{\|}^{h} \\
\times 10^{2}\end{array}$ & $\begin{array}{c}\epsilon_{\perp}^{i} \\
\times 10^{2}\end{array}$ & $E_{h_{w}}^{j}$ & $E_{\bar{h}}^{k}$ & $\begin{array}{c}R_{\perp}^{l} \\
\times 10^{2}\end{array}$ & $\begin{array}{c}\delta^{m} \\
\times 10^{3}\end{array}$ \\
\hline 1.03 & 2.6 & 3.0 & 0.9 & 1.9 & 1.0 & 0.4 & 0.6 & 0.2 & 0.2 & 2.5 & 2.4 & 2 \\
- & - & - & 0.6 & - & 2.1 & 0.3 & 1.2 & 0.1 & - & 5.6 & 2.0 & 1 \\
1.85 & 3.0 & 4.0 & 1.3 & 1.7 & 2.6 & 0.4 & 1.7 & 0.2 & 0.1 & 1.2 & 2.6 & 3 \\
- & - & - & 1.1 & - & 2.7 & 0.4 & 1.8 & 0.2 & - & 1.7 & 3.1 & 3 \\
2.51 & 3.3 & 4.7 & 1.4 & 1.5 & 4.0 & 0.5 & 2.9 & 0.2 & 0.1 & 1.0 & 3.2 & 3 \\
- & - & - & 1.0 & - & 6.0 & 0.5 & 4.3 & 0.3 & - & 2.0 & 4.6 & 2 \\
3.05 & 3.4 & 5.1 & 1.6 & 1.5 & 5.3 & 0.5 & 4.0 & 0.2 & 0.1 & 0.8 & 3.5 & 4 \\
- & - & - & 1.3 & - & 6.0 & 0.5 & 4.5 & 0.3 & - & 1.2 & 4.4 & 3 \\
3.73 & 3.6 & 5.7 & 1.7 & 1.4 & 8.4 & 0.6 & 6.7 & 0.2 & 0.1 & 0.7 & 4.0 & 4 \\
- & - & - & 1.7 & - & 6.6 & 0.5 & 5.3 & 0.2 & - & 0.7 & 4.0 & 4 \\
4.51 & 3.8 & 6.3 & 1.5 & 1.3 & 7.5 & 0.7 & 6.2 & 0.3 & 0.1 & 0.9 & 5.5 & 4 \\
- & - & - & 1.2 & - & 6.3 & 0.7 & 5.3 & 0.4 & - & 1.4 & 7.1 & 3 \\
\hline
\end{tabular}

$\dagger$ For all experiments, $f=1 \mathrm{~s}^{-1}, \Delta \rho=23.87 \pm 0.07 \mathrm{~kg} \mathrm{~m}^{-3}$, and $g^{\prime}=0.23 \mathrm{~m} \mathrm{~s}^{-2}$

${ }^{a}$ Freshwater inflow rate

${ }^{b}$ Internal deformation radius, $l_{D}=\left(2 g^{\prime} q / f^{3}\right)^{1 / 4}$

${ }^{c}$ Buoyant layer depth $(h)$ near the wall, $h_{w}=\sqrt{2 f q / g^{\prime}}$

${ }^{d}$ Average of $h$ at final time of experiment $t_{f}, \bar{h}=q t_{f} / S$ where $S$ is surface area of basin

${ }^{e}$ Kelvin number $K=l_{I} / l_{D}$, where $l_{I}=5 \mathrm{~cm}$ is width of freshwater inlet

${ }^{f}$ Froude number $F_{\|}=U_{\|} / C$, where $U_{\|}$is velocity parallel to the wall and $C=\sqrt{g^{\prime} h_{w}}$

${ }^{g}$ Froude number $F_{\perp}=U_{\perp} / C$, where $U_{\perp}$ is velocity normal to the wall

${ }^{h}$ Rossby number $\epsilon_{\|}=1 / \Delta t_{\|} f$, where $\Delta t_{\|}$is travel time from bulge to corner C1

${ }^{i}$ Rossby number $\epsilon_{\perp}=1 / \Delta t_{\perp} f$, where $\Delta t_{\perp}$ is travel time from $l_{\mathrm{o}}=2 \mathrm{~cm}$ to $l_{\mathrm{o}}=38 \mathrm{~cm}$

${ }^{j}$ Ekman number based on $h_{w}, E_{h_{w}}=2 v / h_{w}^{2} f$

${ }^{k}$ Ekman number based on $\bar{h}, E_{\bar{h}}=2 v / \bar{h}^{2} f$

${ }^{l}$ Reynolds number $R_{\perp}=L_{\perp *}^{2} / \nu \Delta t_{\perp}$, where $L_{\perp *}=(38-2) \times 1.10=40 \mathrm{~cm}$

${ }^{m}$ Aspect ratio $\delta=\bar{h} / L_{\perp}$, where $L_{\perp}=38 \times 1.10=42 \mathrm{~cm}$

dimensionless flow rate $q \times\left(2 f / g^{\prime} h_{0}^{2}\right)$, where $g^{\prime} h_{0}^{2} / 2 f$ is the volume transport of a buoyant flow in thermal wind balance with reduced gravity $g^{\prime}$ and inflow depth $h_{0}$ (Fig. 5). The front speeds are lower than $\sqrt{g^{\prime} h_{0}}$ by two orders of magnitude and tend to increase with the rate of freshwater inflow.

Consider then the coefficient $\alpha_{2}$, which is half the (constant) acceleration of the salinity front. The least-squares estimates of $\alpha_{2}\left(a_{2}\right)$ are normalized by $f \sqrt{g^{\prime} h_{0}} / 2$ and also displayed as a function of the dimensionless flow rate $q \times\left(2 f / g^{\prime} h_{0}^{2}\right)$ (Fig. 6). As expected from the evolution of the front (Fig. 4), the acceleration is negative for all experiments (Fig. 6). Thus, the front decelerates for all values of the rate of freshwater inflow. The deceleration rate increases also in amplitude as the rate of freshwater inflow increases (Fig. 6).

ii. Dimensionless numbers. Dimensionless numbers characterizing the gravity currents in our experiments are estimated in order to compare with bulk properties of buoyant water 


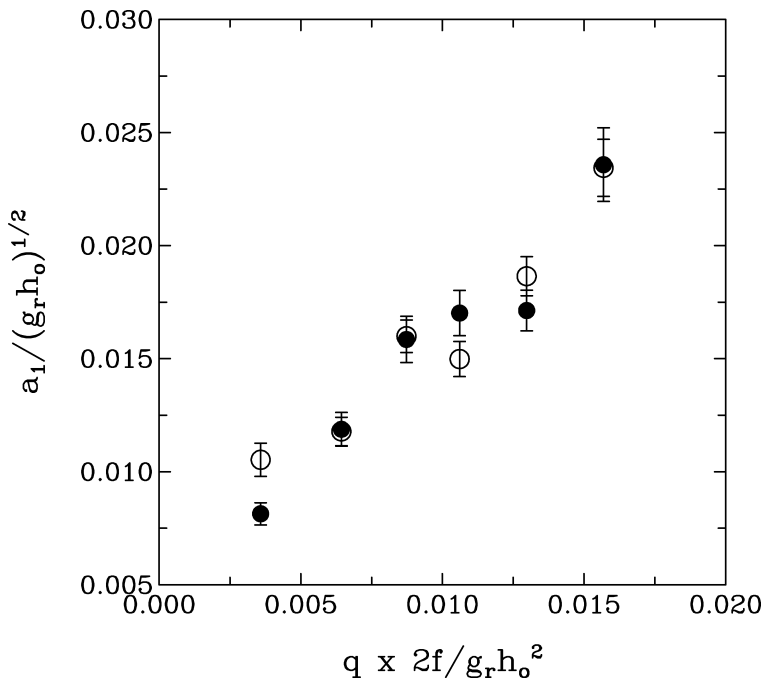

Figure 5. Initial speed of the salinity front as a function of the rate of freshwater inflow. The dimensional speed $a_{1}$ is normalized by $\sqrt{g^{\prime} h_{0}}$, where $g^{\prime}$ is the reduced gravity ( $g_{r}$ in the figure). It is determined for each experiment by quadratic regression of $l$ versus $t$ (note that in the regressions errors in $l$ but not in $t$ are considered). Vertical bars denote uncertainty estimates obtained by regression. The freshwater inflow $q$ is normalized by $g^{\prime} h_{0}^{2} / 2 f$. The uncertainties in freshwater inflow are smaller than the diameter of the circles and are not shown. Results from duplicate experiments in the laboratory are shown by solid and open circles.

discharges into the coastal ocean and to give insight into the dynamics (Table 1). All these numbers vary with the rate of freshwater inflow. The inlet Kelvin number $K$ is the ratio of the width of the buoyant water inlet to the deformation radius. For $K$ of order one or more, rotation should be important for the evolution of the buoyant plume (e.g., Garvine, 1987, 1999). The inlet Kelvin numbers from 1.3 to 1.9 in our experiments (Table 1) are comparable to observational estimates for some buoyant inflows into the coastal ocean. For example, the inlet Kelvin number for the inflow from the Delaware Bay has been estimated to be 1.3 by Garvine (1999) and to be 4 by Avicola and Huq (2003a). A Kelvin number has also been defined as the ratio of the across-shore scale of the buoyant current to the deformation radius and estimated to lie in the range from 0.1 to 10.0 for twelve coastal discharges (see Garvine (1995); Table 1). The across-shore scale of the buoyant current reaches a value much larger than $l_{D}$ in all our experiments (Fig. 4). However, comparing the resulting Kelvin numbers to observational estimates for coastal discharges is difficult since the across-shore scale of the buoyant current varies significantly with time in our experiments, preventing a stable definition.

The Froude number for the along-shore flow, $F_{\|}$, is the ratio of along-shore velocity $U_{\|}$ to phase speed of internal gravity wave, $C$ (e.g., Garvine, 1995). For $F_{\|} \ll 1$, the advective acceleration is negligible compared to the pressure gradient in the momentum balance for 


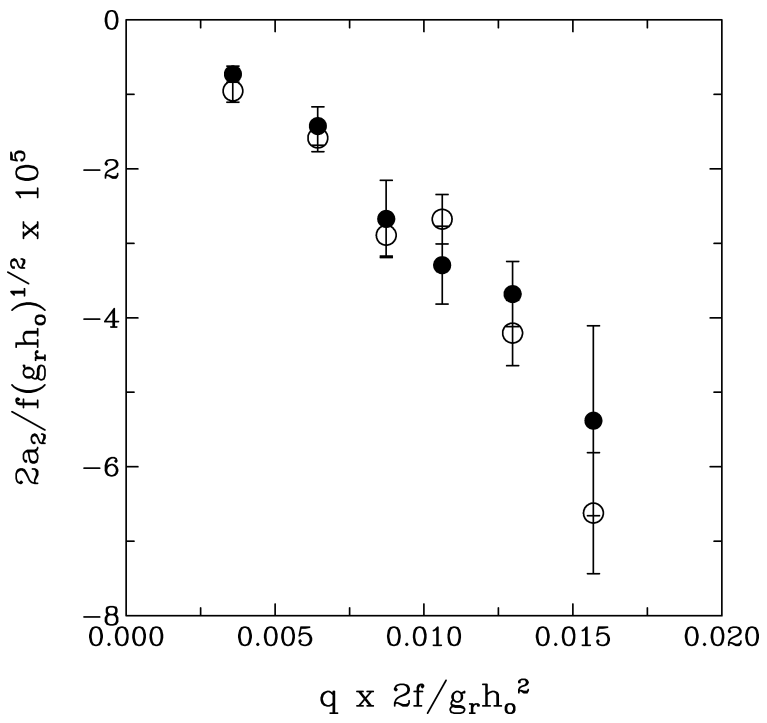

Figure 6. Acceleration of the salinity front as a function of the rate of freshwater inflow. The dimensional acceleration $2 a_{2}$ is normalized by $f \sqrt{g^{\prime} h_{0}}$, where $g^{\prime}$ is the reduced gravity ( $g_{r}$ in the figure). The acceleration $2 a_{2}$ is determined for each experiment by quadratic regression of $l$ versus $t$ (note that in the regressions errors in $l$ but not in $t$ are considered). Vertical bars denote uncertainty estimates obtained by regression. The freshwater inflow $q$ is normalized by $g^{\prime} h_{0}^{2} / 2 f$. The uncertainties in freshwater inflow are smaller than the diameter of the circles and are not shown. Results from duplicate experiments in the laboratory are shown by solid and open circles.

the along-shore flow. For our experiments, $U_{\|}$is taken as the average velocity of the buoyant water from the freshwater inlet to corner $\mathrm{C} 1$ along the center of the wall. Thus, $U_{\|}$is the ratio $l_{\|} / \Delta t_{\|}$, where $l_{\|}=58-12.5=45 \mathrm{~cm}$ is the distance from the center of the inlet to $\mathrm{C} 1$ and $\Delta t_{\|}$is the difference between the time when buoyant water exits the bulge and the time when it reaches $\mathrm{C}$. Note that only the time when buoyant water first appears in the basin $\left(t_{0}\right)$ and the times when the salinity front reaches different apparent distances $l_{\mathrm{o}}$ from the wall $\left(t_{l_{0}=2 \mathrm{~cm}}=t_{1}, \ldots\right)$ have been measured in our experiments. Thus, $\Delta t_{\|}$is taken as the difference $t_{1}-t_{0}$ corrected for the time spent by buoyant water in the bulge $\left(\Delta t_{b}\right)$ and for the transit time of the salinity front from corner $\mathrm{C} 1$ at the wall to the apparent position $l_{\mathrm{o}}=2 \mathrm{~cm}\left(\Delta t_{w}\right)$. The time interval $\Delta t_{b}$ is estimated by assuming that the circulation of buoyant water within the bulge is in gradient wind balance (e.g., Yankovsky and Chapman, 1997) and that buoyant water circulates within the bulge over a distance equal to one half its circumference. Experimental support for a gradient-wind balance in the bulge is provided by Horner-Devine et al. (2006). From the two assumptions above,

$$
\Delta t_{b}=\frac{\pi}{f} \frac{3 g^{\prime} h_{0}+v_{i}^{2}}{2 g^{\prime} h_{0}+v_{i}^{2}},
$$


where $v_{i}$ is the velocity of the freshwater inflow (i.e., the freshwater inflow rate $q$ divided by the surface area of the freshwater inlet). In our experiments, the product $g^{\prime} h_{0}$ always exceeds $v_{i}^{2}$ by several orders of magnitude, implying that to a good approximation $\Delta t_{b}$ is equal to $3 \pi / 2 f \approx 5 \mathrm{~s}$. The transit time of the salinity front from corner $\mathrm{C} 1$ to $l_{\mathrm{o}}=2 \mathrm{~cm}$ is computed from $\Delta t_{w}=(0.02 \times 1.10) / a_{1}$ where $a_{1}$ is speed of the front near the wall (Section $\left.2 \mathrm{bi}\right)$. The phase speed $C$, on the other hand, is computed from $C=\sqrt{g^{\prime} h_{w}}$, where $h_{w}=\sqrt{2 f q / g^{\prime}}$ is the depth of the buoyant layer near the wall. In our experiments, $h_{w}$ ranges from $3.0 \mathrm{~mm}$ for $q=1.03 \mathrm{ml} \mathrm{s}^{-1}$ to $6.3 \mathrm{~mm}$ for $q=4.51 \mathrm{ml} \mathrm{s}^{-1}$ (Table 1), which corresponds to $C$ equal to $2.6 \mathrm{~cm} \mathrm{~s}^{-1}$ and $3.8 \mathrm{~cm} \mathrm{~s}^{-1}$, respectively. Hence the Froude number $F_{\|}$varies from 0.10 to 0.84 (Table 1), indicating that the motion of the current nose along the wall is subcritical to near-critical. This range is close to the range of observational estimates from 0.1 to 1.0 for eleven of the twelve coastal discharges considered by Garvine (1995) (his Table 1).

Finally, a Rossby number can be defined for the along-shore flow as $\epsilon_{\|}=1 / \Delta t_{\|} f$. The resulting values from 0.006 to 0.067 (Table 1) suggest that the Coriolis acceleration dominates the total acceleration of the buoyant current along the wall. Observational estimates for coastal discharges from 0.002 to 5 are computed from parameter estimates reported in Table 1 of Garvine (1995) (specifically, $\epsilon_{\|}$is equivalent to $\gamma F / K$, where $\gamma, F, K$ are parameter estimates listed in his Table 1). Our experimental values bracket observational estimates for five of the twelve coastal discharges considered by this author.

Other dimensionless numbers characterizing the gravity currents in our experiments are also calculated (Table 1). Whereas observational estimates of these numbers for coastal discharges are unknown to us, they are useful in providing insight into the dynamics of the migration of the salinity front away from the wall. The Froude number for the front migration is $F_{\perp}=U_{\perp} / C$, where $U_{\perp}$ is the velocity in the direction normal to the wall. It is estimated from $F_{\perp}=a_{1} / \sqrt{g^{\prime} h_{w}}$, which gives values ranging from 0.03 to 0.07 (Table 1 ). As expected, the slow movement of the salinity front is strongly subcritical. The Rossby number for the front migration is computed from $\epsilon_{\perp}=1 / \Delta t_{\perp} f$, where $\Delta t_{\perp}$ is the travel time of the front from the apparent positions $l_{\mathrm{o}}=2 \mathrm{~cm}$ to $l_{\mathrm{o}}=38 \mathrm{~cm}$. The small values of $\epsilon_{\perp}$ (from 0.001 to 0.004 ; Table 1) suggest that the total acceleration of the flow in the direction normal to the wall is also dominated by the Coriolis acceleration. Two different Ekman numbers are computed: one based on the depth of the buoyant layer near the wall $\left(E_{h_{w}}=2 v / h_{w}^{2} f\right)$ and one based on the average depth of this layer in the basin $\left(E_{\bar{h}}=2 v / \bar{h}^{2} f\right)$. The resulting values of $E_{h_{w}}$ and $E_{\bar{h}}$ are, respectively of order 0.1 and 1 (Table 1), suggesting that vertical friction is not a negligible term in the momentum balance of the layer. Finally, the Reynolds number for the front migration is computed from $R_{\perp}=l_{\perp}^{2} / \nu \Delta t_{\perp}$, where $l_{\perp}=(38-2) \times 1.10=40 \mathrm{~cm}$ is the actual distance covered by the front during $\Delta t_{\perp}$. The estimated values of $R_{\perp} \gg 1$ (Table 1) suggest that horizontal advective acceleration is more important than horizontal friction.

In summary, the Kelvin, Froude, and Rossby numbers characterizing the gravity currents in our experiments compare favorably with observational estimates for buoyant discharges into the coastal ocean. The widening of the currents or, equivalently, the migration of the 
salinity fronts away from the wall appears to be governed by a dynamical balance dominated by the Coriolis acceleration, horizontal pressure gradient, and vertical friction.

\section{Theory}

In this section, the movement of the salinity front observed in the laboratory is given a dynamical interpretation. We consider the evolution of a thin layer of fluid on top of denser fluid that is infinitely deep. Both layers have constant density and are subject to uniform rotation. Although the free surface has a (variable) slope in our experiments, this slope is assumed to have a negligible influence and the free surface is flat in the theory (for a discussion of this influence see Section 5a). On the other hand, the layer interface varies with space and time, and it is allowed to meet the free surface.

The equations of motion for each layer are, in Cartesian coordinates (e.g., Pedlosky, 1987),

$$
\begin{aligned}
\frac{\partial u}{\partial t}+(\mathbf{u} \cdot \nabla) u-f v & =-\frac{1}{\rho} \frac{\partial \tilde{p}}{\partial x}+\nu \nabla^{2} u, \\
\frac{\partial v}{\partial t}+(\mathbf{u} \cdot \nabla) v+f u & =-\frac{1}{\rho} \frac{\partial \tilde{p}}{\partial y}+\nu \nabla^{2} v, \\
\frac{\partial w}{\partial t}+(\mathbf{u} \cdot \nabla) w & =-\frac{1}{\rho} \frac{\partial \tilde{p}}{\partial z}+\nu \nabla^{2} w, \\
\nabla \cdot \mathbf{u} & =0 .
\end{aligned}
$$

Here $\mathbf{u}=(u, v, w)$ is the velocity with components in the directions $(x, y, z),(x, y)$ are the horizontal directions, $z$ is the vertical direction, $t$ is time, $f$ the Coriolis parameter, $\tilde{p}$ the sum $p+\rho g z, p$ the pressure, $\rho$ the density, $g$ the acceleration due to gravity, and $v$ the kinematic viscosity. The nabla operator is $\nabla=\hat{x} \partial / \partial x+\hat{y} \partial / \partial y+\hat{z} \partial / \partial z$, where $(\hat{x}, \hat{y}, \hat{z})$ are unit vectors. The free surface is at $z=0$ and $z$ is positive vertically upwards.

The equations of motion $(4 \mathrm{a}-4 \mathrm{~d})$ are expressed in dimensionless form in order to clarify the nature of the dynamical approximations to be made. We set $t=T t^{\prime},(x, y)=L\left(x^{\prime}, y^{\prime}\right)$, $z=D z^{\prime},(u, v)=U\left(u^{\prime}, v^{\prime}\right), w=(D / L) U w^{\prime}$, and $\tilde{p}=\rho f U L \tilde{p}^{\prime}$, where the primed quantities are dimensionless and $T, L, D, U$ are scales for time, length, depth, and horizontal velocity, respectively. Eqs. (4a-4d) then take the form,

$$
\begin{aligned}
\epsilon_{T} \frac{\partial u^{\prime}}{\partial t^{\prime}}+\epsilon \mathbf{u}^{\prime} \cdot \nabla^{\prime} u^{\prime}-v^{\prime} & =-\frac{\partial \tilde{p}^{\prime}}{\partial x^{\prime}}+\delta^{2} \frac{E}{2}\left(\frac{\partial^{2} u^{\prime}}{\partial x^{\prime 2}}+\frac{\partial^{2} u^{\prime}}{\partial y^{\prime 2}}\right)+\frac{E}{2} \frac{\partial^{2} u^{\prime}}{\partial z^{\prime 2}} \\
\epsilon_{T} \frac{\partial v^{\prime}}{\partial t^{\prime}}+\epsilon \mathbf{u}^{\prime} \cdot \nabla^{\prime} v^{\prime}+u^{\prime} & =-\frac{\partial \tilde{p}^{\prime}}{\partial y^{\prime}}+\delta^{2} \frac{E}{2}\left(\frac{\partial^{2} v^{\prime}}{\partial x^{\prime 2}}+\frac{\partial^{2} v^{\prime}}{\partial y^{\prime 2}}\right)+\frac{E}{2} \frac{\partial^{2} v^{\prime}}{\partial z^{\prime 2}} \\
\delta^{2} \epsilon_{T} \frac{\partial w^{\prime}}{\partial t^{\prime}}+\delta^{2} \epsilon \mathbf{u}^{\prime} \cdot \nabla^{\prime} w^{\prime} & =-\frac{\partial \tilde{p}^{\prime}}{\partial z^{\prime}}+\delta^{4} \frac{E}{2}\left(\frac{\partial^{2} w^{\prime}}{\partial x^{\prime 2}}+\frac{\partial^{2} w^{\prime}}{\partial y^{\prime 2}}\right)+\delta^{2} \frac{E}{2} \frac{\partial^{2} w^{\prime}}{\partial z^{\prime 2}}, \\
\nabla^{\prime} \cdot \mathbf{u}^{\prime} & =0 .
\end{aligned}
$$


Here $\epsilon_{T}=1 /(f T)$ is a Rossby number based on the local acceleration, $\epsilon=U /(f L)$ is a Rossby number based on the advective acceleration, $E=2 v /\left(D^{2} f\right)$ is the Ekman number, and $\delta=D / L$ is the aspect ratio of the motion. Clearly, $\mathbf{u}^{\prime}=\left(u^{\prime}, v^{\prime}, w^{\prime}\right)$ and $\nabla^{\prime}=\hat{x} \partial / \partial x^{\prime}+\hat{y} \partial / \partial y^{\prime}+\hat{z} \partial / \partial z^{\prime}$.

The movement of the salinity front that is observed in the laboratory is characterized by small Rossby number and small aspect ratio, so that $\epsilon_{\perp}=\mathrm{O}\left(10^{-3}\right), \delta^{2} E_{h_{w}}=\mathrm{O}\left(10^{-4}\right)$, and $\delta^{2} E_{\bar{h}}<\mathrm{O}\left(10^{-2}\right)$ (Table 1). Consequently, terms of order $\left(\epsilon_{T}, \epsilon, \delta^{2} E\right)$ and higher are omitted from equations (5a-5d). The resulting equations contain the Ekman number $E$ as the sole parameter. Note that the value of $E=2 v /\left(D^{2} f\right)$ is not known precisely (there is no unique definition of the vertical scale $D$ ), whereas the dimensional equations contain parameters that can all be determined unambiguously from experimental conditions. Accordingly, the approximate equations are considered below in dimensional form in order to compare quantitatively predictions from the theory with our experimental results. Variables in the upper (lower) layer are designated with subscript 1 (2).

\section{a. Upper layer}

The equations of motion for the upper layer are:

$$
\begin{aligned}
-f v_{1} & =-\frac{1}{\rho_{1}} \frac{\partial \tilde{p}_{1}}{\partial x}+v \frac{\partial^{2} u_{1}}{\partial z^{2}}, \\
+f u_{1} & =-\frac{1}{\rho_{1}} \frac{\partial \tilde{p}_{1}}{\partial y}+v \frac{\partial^{2} v_{1}}{\partial z^{2}}, \\
0 & =-\frac{1}{\rho_{1}} \frac{\partial \tilde{p}_{1}}{\partial z} \\
\frac{\partial u_{1}}{\partial x}+\frac{\partial v_{1}}{\partial y}+\frac{\partial w_{1}}{\partial z} & =0 .
\end{aligned}
$$

It is convenient to express these equations in terms of the depth of the upper layer, $h=$ $h(x, y, t)$. First, continuity of pressure at the interface between the two layers at depth $z=-h$ implies that

$$
\rho_{1} g h+\tilde{p}_{1}=\rho_{2} g h+\tilde{p}_{2},
$$

since $\tilde{p}$ in each layer does not vary with depth. The pressure difference between the lower layer and the upper layer is thus given by

$$
\tilde{p}_{2}-\tilde{p}_{1}=-\left(\rho_{2}-\rho_{1}\right) g h .
$$

The horizontal gradient of $\tilde{p}$ in the lower layer is zero if the horizontal components of velocity in this layer, which is infinitely deep, are zero at $z=-\infty$. Indeed, in this case, the horizontal gradient of $\tilde{p}$ vanishes at $z=-\infty$ and, since $\partial \tilde{p} / \partial z$ is nil, this gradient 
must vanish at all depths in the lower layer. Hence, with $\partial \tilde{p}_{2} / \partial x$ and $\partial \tilde{p}_{2} / \partial y$ both equal to zero, the horizontal pressure gradient in the upper layer can be expressed in terms of the horizontal gradient of $h$, e.g,

$$
-\frac{1}{\rho_{1}} \frac{\partial \tilde{p}_{1}}{\partial x}=-g^{\prime} \frac{\partial h}{\partial x}
$$

where $g^{\prime}=g\left(\rho_{2}-\rho_{1}\right) / \rho_{1}$ is the reduced gravity. A similar expression holds for the pressure gradient component in the $y$-direction. Second, the incompressibility condition (6d) is integrated from the layer interface at $z=-h$ to the free surface at $z=0$,

$$
w_{1}(0)-w_{1}(-h)=-\int_{-h}^{0}\left[\frac{\partial u_{1}}{\partial x}+\frac{\partial v_{1}}{\partial y}\right] d z
$$

The following kinematical conditions are imposed,

$$
\begin{array}{ll}
w_{1}=0 & \text { at } z=0, \\
w_{1}=-\left[\frac{\partial h}{\partial t}+u_{1} \frac{\partial h}{\partial x}+v_{1} \frac{\partial h}{\partial y}\right] & \text { at } z=-h(x, y, t) .
\end{array}
$$

The first condition follows from the free surface being flat and the second condition states that the interface is a material surface. Inserting both conditions in (10) gives

$$
\frac{\partial h}{\partial t}+u_{1}(-h) \frac{\partial h}{\partial x}+v_{1}(-h) \frac{\partial h}{\partial y}=-\frac{\partial}{\partial x} \int_{-h}^{0} u_{1} d z+u_{1}(-h) \frac{\partial h}{\partial x}-\frac{\partial}{\partial y} \int_{-h}^{0} v_{1} d z+v_{1}(-h) \frac{\partial h}{\partial y},
$$

upon application of the Leibniz rule for the derivation of an integral with variable limits. The equations of motion for the upper layer can then be cast in terms of $h$,

$$
\begin{gathered}
-f v_{1}=-g^{\prime} \frac{\partial h}{\partial x}+v \frac{\partial^{2} u_{1}}{\partial z^{2}}, \\
+f u_{1}=-g^{\prime} \frac{\partial h}{\partial y}+v \frac{\partial^{2} v_{1}}{\partial z^{2}}, \\
\frac{\partial h}{\partial t}+\frac{\partial}{\partial x} \int_{-h}^{0} u_{1} d z+\frac{\partial}{\partial y} \int_{-h}^{0} v_{1} d z=0 .
\end{gathered}
$$

Note that the statement of volume conservation (13c) is nonlinear owing to the variable limit $h=h(x, y, t)$ of the integrals. 
The momentum equations (13a-13b) are the equations of the Ekman problem for a fluid with uniform density. Here these equations are solved with a condition of no stress at the surface,

$$
\nu \frac{\partial u_{1}}{\partial z}=\nu \frac{\partial v_{1}}{\partial z}=0 \quad \text { at } z=0 .
$$

The solutions of (13a-13b) which satisfy these conditions are

$$
\begin{aligned}
& u_{1}=A_{1} \cos \frac{z}{\delta} \cosh \frac{z}{\delta}-B_{1} \sin \frac{z}{\delta} \sinh \frac{z}{\delta}+u_{g}, \\
& v_{1}=A_{1} \sin \frac{z}{\delta} \sinh \frac{z}{\delta}+B_{1} \cos \frac{z}{\delta} \cosh \frac{z}{\delta}+v_{g},
\end{aligned}
$$

where $\left(A_{1}, B_{1}\right)$ are real constants, $\delta=\sqrt{2 v / f}$ is the Ekman layer depth, and $\left(u_{g}, v_{g}\right)=$ $\left(g^{\prime} / f\right)(-\partial h / \partial y, \partial h / \partial x)$ are the geostrophic velocities.

\section{b. Lower layer}

The same approximations are made as for the upper layer, with the additional restriction that the horizontal components of the pressure gradient are zero (see Section 3a). Hence, the horizontal momentum equations for the lower layer are simply

$$
\begin{aligned}
& -f v_{2}=v \frac{\partial^{2} u_{2}}{\partial z^{2}}, \\
& +f u_{2}=v \frac{\partial^{2} v_{2}}{\partial z^{2}} .
\end{aligned}
$$

These equations are solved with the condition that the horizontal velocity components vanish at depth,

$$
u_{2}=v_{2}=0 \quad \text { at } z=-\infty .
$$

The solutions of (16a-16b) which satisfy these conditions are

$$
\begin{aligned}
& u_{2}=A_{2} e^{z / \delta} \cos \frac{z}{\delta}-B_{2} e^{z / \delta} \sin \frac{z}{\delta}, \\
& v_{2}=A_{2} e^{z / \delta} \sin \frac{z}{\delta}+B_{2} e^{z / \delta} \cos \frac{z}{\delta},
\end{aligned}
$$

where $\left(A_{2}, B_{2}\right)$ are also real constants.

\section{c. Matching conditions}

The horizontal velocity in each layer is provided by expressions (15a-15b) and (18a18b). These expressions contain four constants $\left(A_{1}, B_{1}, A_{2}, B_{2}\right)$ that are determined by conditions imposed at the interface between the upper layer and the lower layer. More 
specifically, continuity of horizontal velocity and of vertical stress is invoked at the interface between the two layers,

$$
\begin{aligned}
u_{1} & =u_{2} & \text { at } z=-h(x, y, t), \\
v_{1} & =v_{2} & - \\
\nu \frac{\partial u_{1}}{\partial z} & =\nu \frac{\partial u_{2}}{\partial z} & - \\
\nu \frac{\partial v_{1}}{\partial z} & =\nu \frac{\partial v_{2}}{\partial z} & -
\end{aligned}
$$

Applying these conditions to (15a-15b) and (18a-18b) leads to a system of four algebraic equations which are linear in $A_{1}, B_{1}, A_{2}, B_{2}$ and from which these constants can be determined:

$$
\begin{aligned}
& A_{1}=-e^{-h / \delta}\left(u_{g} \cos \frac{h}{\delta}+v_{g} \sin \frac{h}{\delta}\right), \\
& B_{1}=+e^{-h / \delta}\left(u_{g} \sin \frac{h}{\delta}-v_{g} \cos \frac{h}{\delta}\right),
\end{aligned}
$$

and

$$
\begin{aligned}
& A_{2}=u_{g} \cos \frac{h}{\delta} \sinh \frac{h}{\delta}-v_{g} \sin \frac{h}{\delta} \cosh \frac{h}{\delta}, \\
& B_{2}=u_{g} \sin \frac{h}{\delta} \cosh \frac{h}{\delta}+v_{g} \cos \frac{h}{\delta} \sinh \frac{h}{\delta} .
\end{aligned}
$$

Inserting (20a-20b) and (21a-21b) into (15a-15b) and (18a-18b) allows one to express the horizontal velocity in the upper and lower layers solely in terms of the upper layer depth and of its horizontal variations.

\section{d. Horizontal velocities}

We consider the vertical distribution of the horizontal velocity components in both layers for varying values of $h / \delta$. Since rotation is uniform, no particular horizontal direction is dynamically significant; the axes $(x, y)$ of the Cartesian reference frame are arbitrary and can been chosen for convenience. For example, the $x$-axis can be aligned in the direction of the horizontal pressure gradient, so $\partial h / \partial y$ vanishes and the expressions for the horizontal velocity in the two layers take a simpler form. In this Section, $\partial h / \partial y$ is taken as zero without loss of generality, and the horizontal velocity components are normalized by the geostrophic value $\left(g^{\prime} \partial h / \partial x\right) / f$.

Consider first the case where $h / \delta=10$ (Fig. 7a). Over most of the upper layer, the velocity component $u_{1}$ has a very small amplitude as there is no pressure gradient to balance geostrophic motion in the $x$-direction, whereas the velocity component $v_{1}$ is close to 

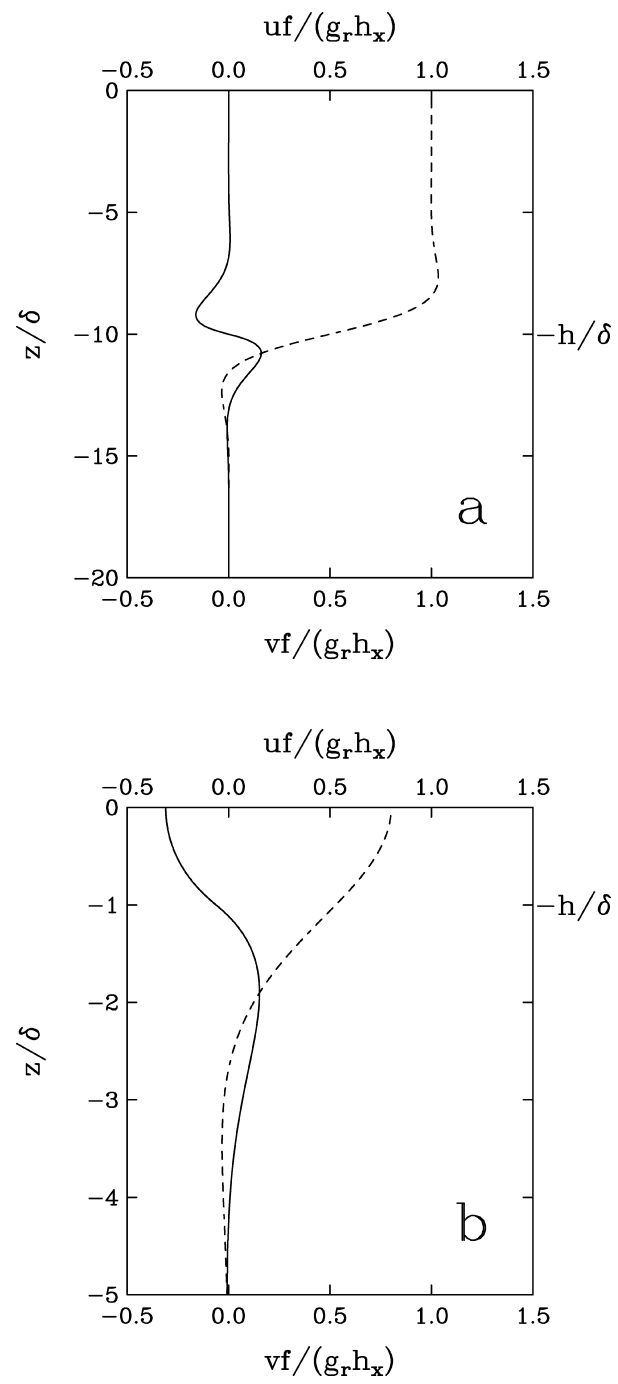

Figure 7. Solutions of the two-layer theory. Vertical distribution of the horizontal velocity components $u$ (solid line) and $v$ (long dashed) for the cases where $h / \delta=10$ (a) and $h / \delta=1$ (b). The two components are normalized by $\left(g^{\prime} \partial h / \partial x\right) / f$, where $g^{\prime}$ is the reduced gravity $\left(g_{r}\right.$ in the figure).

$\left(g^{\prime} \partial h / \partial x\right) / f$. In contrast, near the interface, both $u_{1}$ and $v_{1}$ show large variations. In this region, friction becomes important in the momentum balance. Thus, $\nu \partial^{2} u_{1} / \partial z^{2}$ balances the pressure gradient and $\nu \partial^{2} v_{1} / \partial z^{2}$ balances the Coriolis acceleration of the flow in the $x$-direction, leading to an Ekman spiral (cf. upper branch of solid line in Fig. 8). In the deep layer, where no horizontal gradient of pressure occurs, friction balances the Coriolis acceleration over the whole layer. The stress exerted by motion in the upper layer is 


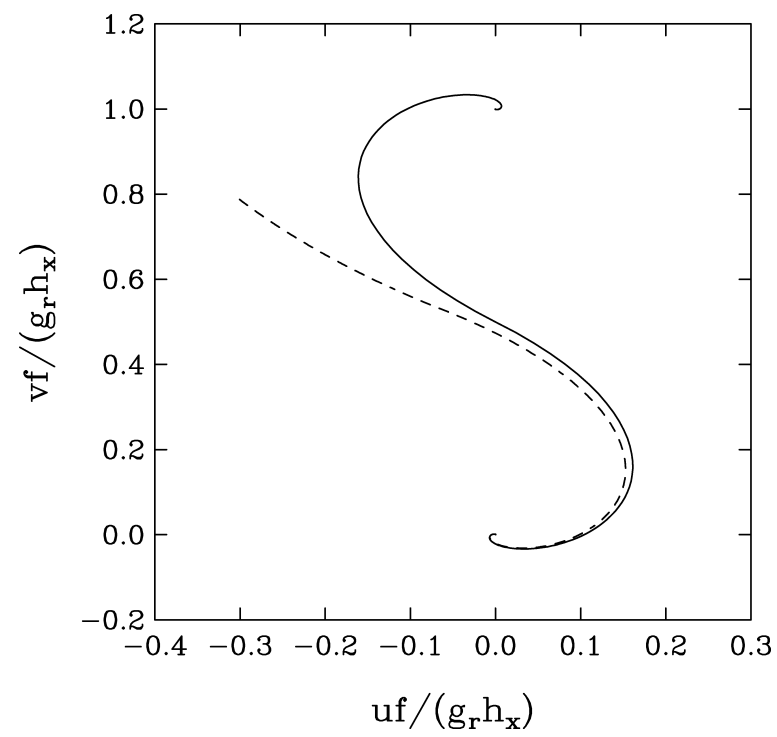

Figure 8. Solutions of the two-layer theory. Velocity component $v$ versus velocity component $u$ for the cases where $h / \delta=10$ (solid line) and $h / \delta=1$ (dashed). The two components are normalized by $\left(g^{\prime} \partial h / \partial x\right) / f$.

communicated to the lower layer. Motion is to the right of the stress owing to the Coriolis acceleration, leading to another Ekman spiral (cf. lower branch of solid line in Fig. 8).

Consider then two cases where $h / \delta$ is much smaller. For $h / \delta=1$, the vertical variations in the horizontal velocity components are relatively smooth in both layers (Fig. 7b). Here friction influences the momentum balance in regions far from the interface between the two layers. An Ekman spiral is present only in the deep layer (dashed line in (Fig. 8). For $h / \delta=0.1$, the horizontal velocity components are nearly uniform in the upper layer and their amplitudes decrease very slowly with depth (not shown).

\section{e. Governing equation for $h$}

A governing equation for $h$ is obtained by inserting the expressions for $u_{1}, v_{1}(15 \mathrm{a}-15 \mathrm{~b})$, where $A_{1}, B_{1}$ are provided by (20a-20b), into the statement of volume conservation (13c). Details are reported in Appendix B. Interestingly, the equation for $h$ can then be written as a diffusion equation,

$$
\frac{\partial h}{\partial t}=\frac{\partial}{\partial x}\left(\kappa_{s} \frac{\partial h}{\partial x}\right)+\frac{\partial}{\partial x}\left(\kappa_{a} \frac{\partial h}{\partial y}\right)+\frac{\partial}{\partial y}\left(-\kappa_{a} \frac{\partial h}{\partial x}\right)+\frac{\partial}{\partial y}\left(\kappa_{s} \frac{\partial h}{\partial y}\right),
$$

where the effective diffusivites $\kappa_{s}$ and $\kappa_{a}$ depend on $h$,

$$
\kappa_{s}=+\kappa_{0}\left\{1-e^{-2 h / \delta}\left(\sin \frac{2 h}{\delta}+\cos \frac{2 h}{\delta}\right)\right\},
$$




$$
\kappa_{a}=-\kappa_{0}\left\{1+e^{-2 h / \delta}\left(\sin \frac{2 h}{\delta}-\cos \frac{2 h}{\delta}\right)-4 \frac{h}{\delta}\right\}
$$

with $\kappa_{0}=g^{\prime} \delta /(4 f)$ being a constant factor. The governing equation for $h$ can be written more compactly as

$$
\frac{\partial h}{\partial t}=\nabla \cdot(\mathbf{K}(h) \nabla h)
$$

where the diffusion tensor $\mathbf{K}(h)$ is the sum of a symmetric part $\mathbf{S}(h)$ proportional to $\kappa_{s}$ and an antisymmetric part $\mathbf{A}(h)$ proportional to $\kappa_{a}$ :

$$
\mathbf{S}(h)=\left(\begin{array}{cc}
\kappa_{s}(h) & 0 \\
0 & \kappa_{s}(h)
\end{array}\right) \quad \text { and } \quad \mathbf{A}(h)=\left(\begin{array}{cc}
0 & \kappa_{a}(h) \\
-\kappa_{a}(h) & 0
\end{array}\right)
$$

Note that Eq. (24) can also be written as an advection-diffusion equation.

The transport that is produced by $\mathbf{S}$ and $\mathbf{A}$ is quite distinct, as shown for any scalar quantity satisfying an equation of the form (24) (Vallis, 2006). The symmetric tensor $\mathbf{S}$ produces a flux of light fluid $-\mathbf{S} \nabla \mathbf{h}$ which is down the gradient of $h$ since $\mathbf{S}$ is positive definite, i.e., $\nabla h \cdot(\mathbf{S} \nabla h)>0$. In contrast, the antisymmetric tensor $\mathbf{A}$ produces a flux of light fluid $-\mathbf{A} \nabla h$ which is perpendicular to the gradient of $h$ since $\nabla h \cdot(\mathbf{A} \nabla h)=0$. From a dynamical point of view, $\mathbf{S}$ represents the effect of vertical friction and $\mathbf{A}$ represents the effect of geostrophic motion, although the two tensors depend formally on both $v$ and $f$. The transport that is produced by $\mathbf{S}$ or $\mathbf{A}$ does not modify the average of the layer depth $h$ in a closed basin along which the normal derivative of $h$ vanishes, i.e., each tensor is separately volume-conserving. On the other hand, the transport induced by $\mathbf{S}$ decreases the basin average of $h^{2}$, whereas the transport induced by $\mathbf{A}$ does not alter this average. Indeed, the 'skew flux' $-\mathbf{A} \nabla h$ is equivalent to the advection by a solenoidal velocity (Vallis, 2006).

Note that, if the layer depth $h$ and its spatial derivatives are continuous, the mixed derivatives $\partial / \partial x(\partial h / \partial y)$ and $\partial / \partial y(\partial h / \partial x)$ are equal. In this case, the two terms with $\kappa_{a}$ in (22) cancel each other. The flux $-\mathbf{A} \nabla h$ has no divergence and the light fluid spreads exclusively in the downgradient direction. Such a situation, however, should not occur in regions where the gradient $\nabla h$ is discontinuous such as a front. Thus, it is expected that the movement of the front is at least partly influenced by the antisymmetric tensor $\mathbf{A}$.

The variations of $\kappa_{s} / \kappa_{0}$ and $\kappa_{a} / \kappa_{0}$ with $h / \delta$ are displayed in Figure 9. The symmetric diffusivity $\kappa_{s}$ increases with $h / \delta$ for small $h / \delta$ and reaches a maximum at $h / \delta=\pi / 2$. In contrast, the antisymmetric diffusivity $\kappa_{a}$ increases monotonically with $h / \delta$. At large $h / \delta$, the increase is about linear due to the secular term $4 h / \delta$ in (23b). In this case, ambient rotation becomes important compared to vertical friction and the light fluid spreads primarily in the direction perpendicular to the gradient of $h$, i.e., perpendicular to the pressure gradient. Note that $\kappa_{a}$ always exceeds $\kappa_{s}$ for $h / \delta>0$. The symmetric and antisymmetric diffusivities tend to the same value as $h / \delta \rightarrow 0$, e.g., as rotation becomes small. 


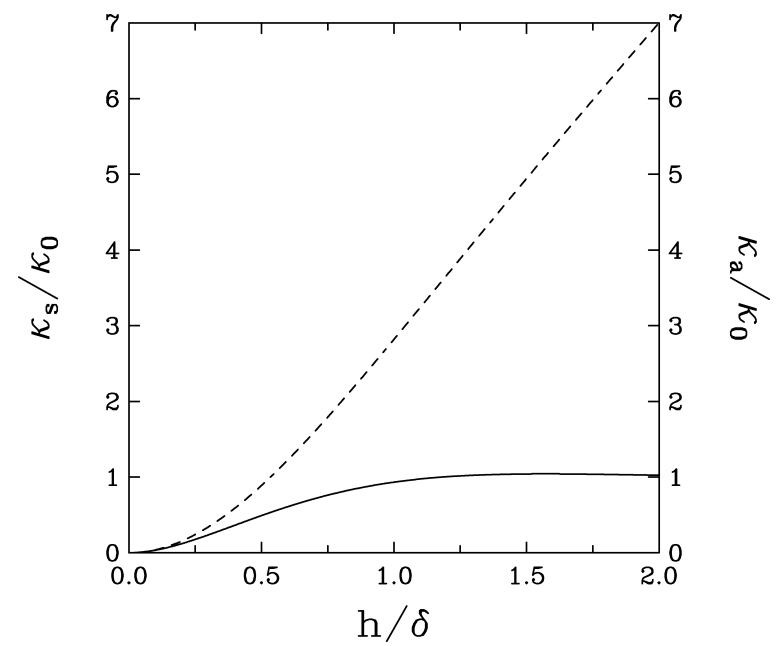

Figure 9. Effective diffusivities for the depth of the upper layer versus the ratio of layer depth to

Ekman layer depth. The symmetric (antisymmetric) diffusivity is shown by the solid (dashed) line.

Each diffusivity is normalized by $\kappa_{0}=g^{\prime} \delta /(4 f)$.

\section{f. Limiting cases $h / \delta \ll 1$ and $h / \delta \gg 1$}

We consider Eq. (22) for $h$ as this depth becomes very thin or very thick compared to the depth of the Ekman layer. For simplicity, it is assumed that the sum of the terms containing $\kappa_{a}$ in (22) vanishes. As we have seen, this assumption is a significant limitation since it should not be valid in regions where $\nabla h$ is discontinuous. Nevertheless, it leads to useful insight into the movement of this layer for contrasting $h / \delta$. For convenience, the equation for the two cases, $h \ll \delta$ and $h \gg \delta$, is derived by scaling the layer depth $h$ with $\delta$, the time $t$ with $f^{-1}$, the horizontal coordinates $(x, y)$ with $\sqrt{g^{\prime} \delta} / f$, and the diffusivity $\kappa_{s}$ with $g^{\prime} \delta / f$. Dimensionless variables are denoted with a prime.

Consider first the case where $h^{\prime} \ll 1$. Expanding (23a) in Taylor series shows that

$$
\kappa_{s}^{\prime}=h^{\prime 2}+\mathrm{O}\left(h^{\prime 3}\right)
$$

The equation for $h^{\prime}$ becomes to order $\mathrm{O}\left(h^{\prime 3}\right)$ a diffusion equation where the effective diffusivity increases quadratically with $h^{\prime}$ :

$$
\frac{\partial h^{\prime}}{\partial t^{\prime}}=\frac{\partial}{\partial x^{\prime}}\left(h^{\prime 2} \frac{\partial h^{\prime}}{\partial x^{\prime}}\right)+\frac{\partial}{\partial y^{\prime}}\left(h^{\prime 2} \frac{\partial h^{\prime}}{\partial y^{\prime}}\right) .
$$

For an instantaneous point source of light fluid, diffusion is expected to be radial everywhere and Eq. (27) is more conveniently expressed in cylindrical coordinates,

$$
\frac{\partial h^{\prime}}{\partial t^{\prime}}=\frac{1}{r^{\prime}} \frac{\partial}{\partial r^{\prime}}\left(r^{\prime} h^{\prime 2} \frac{\partial h^{\prime}}{\partial r^{\prime}}\right)
$$


where $r^{\prime}=\sqrt{x^{\prime 2}+y^{\prime 2}}$ is the radial distance from the source. This equation has been solved analytically for an instantaneous point source in a different physical context (Pattle, 1959). The solution of (28) for a unit source at $\left(r^{\prime}=0, t^{\prime}=0\right)$ and for $h^{\prime}\left(r^{\prime}=0, t^{\prime}=0\right)=1$ is

$$
\begin{array}{ll}
h^{\prime}\left(r^{\prime}, t^{\prime}\right)=\left(\frac{t^{\prime}}{t_{*}^{\prime}}\right)^{-1 / 3}\left(1-\frac{r^{\prime 2}}{r_{*}^{\prime 2}}\right)^{1 / 2} & \text { for } r^{\prime 2} \leq r_{*}^{\prime 2}, \\
h^{\prime}\left(r^{\prime}, t^{\prime}\right)=0 & \text { for } r^{\prime 2} \geq r_{*}^{\prime 2},
\end{array}
$$

where

$$
t_{*}^{\prime}=\frac{1}{4 \pi} \quad \text { and } \quad r_{*}^{\prime 2}=\frac{3}{2 \pi}\left(\frac{t^{\prime}}{t_{*}^{\prime}}\right)^{1 / 3} .
$$

The layer depth $h^{\prime}$ when expressed as a function of the distance $r^{\prime}$ from the source describes one-half of an ellipse. The edge of the layer is at $r^{\prime}= \pm r_{*}^{\prime}$ and moves away from the source at the rate $t^{\prime 1 / 6}$. Note that the pressure gradient (i.e., $\partial h^{\prime} / \partial r^{\prime}$ ) is infinite at $r^{\prime}= \pm r_{*}^{\prime}$, so terms of higher order in (28) should be considered to obtain a physically realistic solution at the edge.

Consider then the case where $h^{\prime} \gg 1$. In this limit expression (23a) shows that

$$
\kappa_{s}^{\prime}=\frac{1}{4}
$$

The equation for $h^{\prime}$ then reduces to a diffusion equation with constant diffusivity:

$$
\frac{\partial h^{\prime}}{\partial t^{\prime}}=\frac{1}{4}\left(\frac{\partial^{2} h^{\prime}}{\partial x^{\prime 2}}+\frac{\partial^{2} h^{\prime}}{\partial y^{\prime 2}}\right)
$$

The solution of (32) for a unit point source at $\left(r^{\prime}=0, t^{\prime}=0\right)$ is well-known (e.g., Crank, 1975),

$$
h^{\prime}\left(r^{\prime}, t^{\prime}\right)=\frac{e^{-r^{\prime 2} / t^{\prime}}}{\pi t^{\prime}}
$$

The layer depth never vanishes and there is no edge sensu stricto for finite $r^{\prime}$ and $t^{\prime}$.

In summary, analysis of the two extreme cases $h / \delta \ll 1$ and $h / \delta \gg 1$ indicates that the evolution of the upper layer will have a fundamentally different character depending on the ratio of the layer depth to the depth of the Ekman layer. If $h$ is small compared to $\delta$, motion along the pressure gradient occurs throughout the upper layer. The layer diffusivity depends on layer depth. In contrast, if $h$ is large compared to $\delta$, motion along the pressure gradient is confined to a region near the interface between the two layers. In this case, the evolution of $h$ is dictated mostly by diffusion with approximately constant diffusivity. 


\section{g. General case for $h / \delta$}

Whereas the exact solutions (29) and (33) are revealing, they do not correspond to conditions in the laboratory where the freshwater source is continuous. Both solutions are further limited in the sense that the first predicts an infinite pressure gradient at the edge and the second does not predict the existence of an edge at all. Here the governing equation for $h$ (22) is solved for a continuous source and arbitrary $h / \delta$ using a numerical method. The method of solution is a finite-difference method that is second-order accurate in time and space, positive definite, and conservative. It solves the governing equation for $h$ in the advective form

$$
\frac{\partial h}{\partial t}+\frac{\partial}{\partial x}(\bar{u} h)+\frac{\partial}{\partial y}(\bar{v} h)=0
$$

where

$$
\begin{aligned}
& \bar{u}=-\frac{\kappa_{s}}{h} \frac{\partial h}{\partial x}-\frac{\kappa_{a}}{h} \frac{\partial h}{\partial y}, \\
& \bar{v}=+\frac{\kappa_{a}}{h} \frac{\partial h}{\partial x}-\frac{\kappa_{s}}{h} \frac{\partial h}{\partial y},
\end{aligned}
$$

are the horizontal components of an effective velocity. This equation is solved on a domain corresponding to the rotating basin in the laboratory. The coordinate $x$ is antiparallel to the adjacent wall and the coordinate $y$ is parallel to the wall. Thus, the velocity component $\bar{u}$ $(\bar{v})$ is normal (parallel) to the wall. Further details about the method of solution are reported in Appendix C.

The initial conditions, boundary conditions, and parameters of equation (34) are all constrained from conditions in the laboratory. Initially, the layer depth $h$ is zero everywhere. The volume fluxes per unit length $(\bar{u} h, \bar{v} h)$ vanish everywhere along the boundary except along the opening made through the wall. There $\bar{u} h=-q / l_{I}$, where $l_{I}=5 \mathrm{~cm}$ is the width of the opening. The parameters governing the transport of buoyant fluid in the theory are the diffusivity factor $\left(\kappa_{0}\right)$ and the Ekman layer depth $(\delta)$ (Eqs. (23a-23b)). They are determined from $g^{\prime}=0.23 \mathrm{~m} \mathrm{~s}^{-2}$ based on density measurements (Section $2 \mathrm{a}$ ), $\nu=10^{-6} \mathrm{~m}^{2} \mathrm{~s}^{-1}$ based on viscosity measurements on pure water at $20^{\circ} \mathrm{C}$ (Weast and Astle, 1981), and $f=1 \mathrm{~s}^{-1}$ corresponding to the turntable rotation rate (Section $2 \mathrm{a}$ ). Consequently, in our numerical solutions, the diffusivity factor $\kappa_{0}$ is $1.0 \times 10^{-4} \mathrm{~m}^{2} \mathrm{~s}^{-1}$ and the Ekman layer depth $\delta$ is $1.4 \mathrm{~mm}$.

\section{Comparison of laboratory results with theory}

In this section, the observations made on the rotating table are compared to numerical solutions of the governing equation for $h$ (34). Numerical solutions are obtained for the different rates of freshwater discharge considered for the laboratory experiments.

We first compare the distributions of buoyant water observed on the turntable and obtained numerically for the case where $q=1.82 \mathrm{ml} \mathrm{s}^{-1}$ (Figs. 10a-10b). The distributions are 



Figure 10. Distribution of buoyant water observed in the laboratory (upper panel) and calculated numerically (lower panel) four minutes after freshwater is introduced into the basin ( $q=$ $1.82 \mathrm{ml} \mathrm{s}^{-1}$ ). Upper panel: buoyant water is shown by the red patch. Lower panel: buoyant water is shown by isopleths of $h / \delta$ with contour interval of 0.5 . The dashed line (isopleth of 0.01 ) approximately coincides with the front.

those four minutes after buoyant water is introduced along the wall. The buoyant water distribution obtained numerically appears similar to that observed on the turntable at the same time (Figs. 10a-10b). As in the laboratory, the buoyant source has led to the formation of a gravity current along the wall flowing toward the arc of the basin and to the development of a density front along the wall. The distance of the front from the wall greatly exceeds 



Figure 11. Experimental measurements and theoretical predictions of the distance of the salinity front from the wall as a function of time for different rates of freshwater inflow (in ml s${ }^{-1}$ ): $1.03 \pm 0.00$ (a), $1.85 \pm 0.00$ (b), $2.51 \pm 0.03$ (c), $3.05 \pm 0.02$ (d), $3.73 \pm 0.04$ (e), and $4.51 \pm 0.08$ (f). The distance from the wall (time) is normalized by $\sqrt{g^{\prime} h_{0}} / f(4 \pi / f)$, where $g^{\prime}$ is the reduced gravity ( $g_{r}$ in the figure). Results from duplicate laboratory experiments are shown by solid and open circles (horizontal and vertical bars denote uncertainty estimates). Numerical solutions of the twolayer theory with $g^{\prime}=0.23 \mathrm{~m} \mathrm{~s}^{-2}\left(0.12 \mathrm{~m} \mathrm{~s}^{-2}\right)$ are shown by solid (dashed) lines. The horizontal dashed line shows the ratio $l_{D} / \sqrt{g^{\prime} h_{0}} / f$ for each experiment, where $l_{D}=\left(2 g^{\prime} q / f^{3}\right)^{1 / 4}$ is the deformation radius.

the deformation radius, as in the laboratory. The gravity current along the wall does not separate from corner $\mathrm{C} 1$, which is consistent with observation.

\section{a. Position of salinity front}

We compare the position of the salinity front at different times as observed in the laboratory and obtained numerically (compare circles with solid line in Figs. 11a-11f). For both the laboratory observations and the numerical solutions, the distance of the front from 


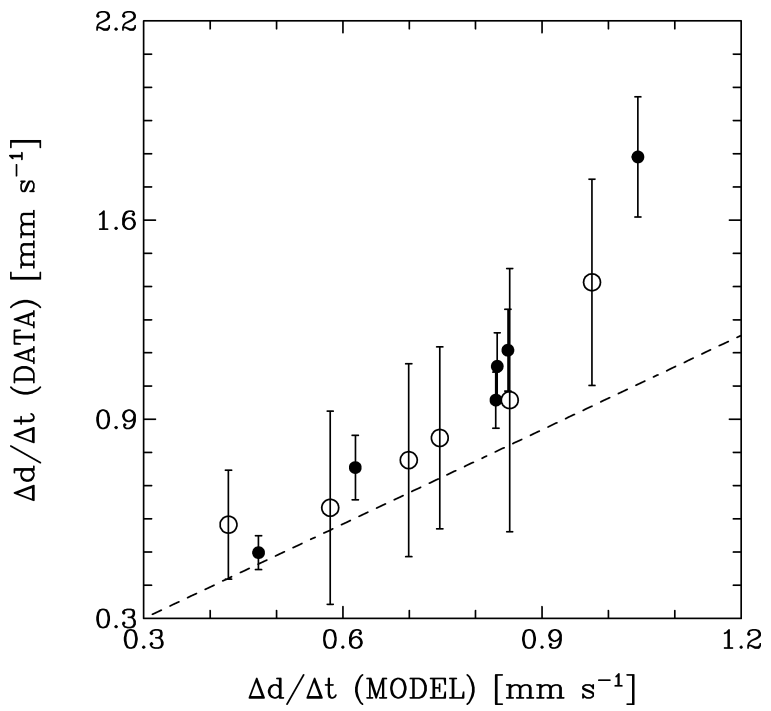

Figure 12. Comparison between the mean speed of the salinity front observed in the laboratory and calculated numerically from theory. Results from duplicate experiments in the laboratory are shown by solid and open circles. Vertical bars denote two standard deviations in the observational estimates. The dashed line (slope $=1$ ) is the line of perfect agreement between the experimental and numerical results.

the wall is determined along the direction normal to the wall and from corner $\mathrm{C} 1$ of the wall. The salinity front as computed numerically migrates persistently away from the wall for all rates of freshwater inflow (Figs. 11a-11f). For each rate of freshwater inflow, the front reaches a distance from the wall of several deformation radii and moves at a speed decreasing with time, which is qualitatively consistent with observation. For the four largest inflow rates, the numerical solution agrees closely with laboratory results with only a few exceptions (Figs. 11c-11f). On the other hand, the distance of the front from the wall as determined numerically exceeds systematically the observed distance for the two smallest rates of freshwater inflow (Figs. 11a-11b).

\section{b. Mean speed of salinity front}

We also compare the mean speed of the salinity front from the laboratory observations and the numerical solutions. The mean speed of the front is defined as $\Delta d / \Delta t=\left(l_{f}-l_{i}\right) /\left(t_{f}-\right.$ $\left.t_{i}\right)$, where $l_{i}=2 \times 1.10 \mathrm{~cm}\left(l_{f}=38 \times 1.10 \mathrm{~cm}\right)$ is the initial (final) recorded distance from the wall. The uncertainty (standard deviation) in the observed mean speed is calculated by propagating the uncertainties in the initial and final distances and the uncertainties in the initial and final times, assuming no correlation between the uncertainties. The difference between the predicted and observed mean speeds is less than two standard deviations for half of the experiments (Fig. 12). The mean speeds from the numerical solutions are smaller 


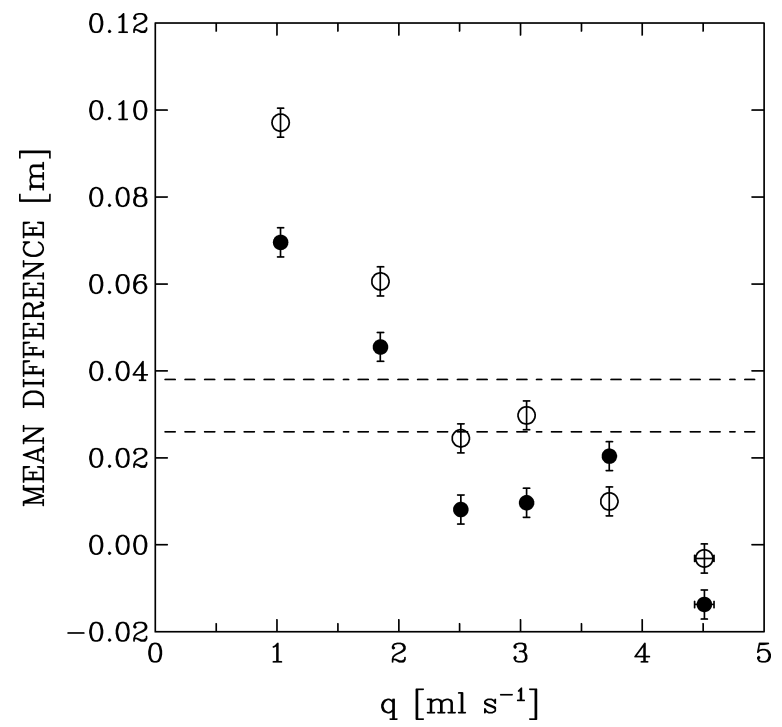

Figure 13. Difference between the distance of the salinity front from the wall predicted by theory and observed in the laboratory. The difference is the average for the ten experimental distances from the wall and is shown as a function of the rate of freshwater inflow. Results for duplicate experiments in the laboratory are shown by solid and open circles. Horizontal and vertical bars denote uncertainty estimates (the uncertainties in freshwater inflow rate for $q \leq 3.73 \mathrm{ml} \mathrm{s}^{-1}$ are smaller than the diameter of the circles and are not shown). The horizontal dashed lines show the range of the deformation radius in the experiments.

than the observed ones for all experiments, suggesting the presence of a bias in the solutions, in the observational estimates, or in both.

\section{Discussion}

In this section, the differences between the experimental results and the numerical solutions of the movement of the salinity front are given some consideration. The most conspicuous difference is the overestimation of the distances of the front from the wall which are predicted for the smallest rates of freshwater inflow (Figs. 11a-11b). The overestimation is reduced for higher inflow rates (Figs. 11c-11f). To better illustrate this, the mean difference between the predicted and observed position of the front is computed for each experiment and plotted against $q$ (Fig. 13). The mean difference decreases from 7-10 cm for $q=1.03 \mathrm{ml} \mathrm{s}^{-1}$ to less than $2 \mathrm{~cm}$ for $q=4.51 \mathrm{ml} \mathrm{s}^{-1}$. Another difference consists in the underestimation of the mean speed at which the front moves away from the wall over the course of the experiments (Fig. 12).

The differences described above are likely due to approximation(s) in the theory, although systematic errors in the laboratory and in the numerical method to solve Eq. (34) cannot be excluded. Below several approximations in the theory are discussed, with emphasis on 
their possible contribution to the differences. Approximations which appear unlikely to contribute are considered first, followed by those whose effects on our experimental results are more difficult to establish.

\section{a. Variation of free surface}

Whereas the free surface is assumed to be flat in theory (11a), rotation produces a radial (parabolic) variation of the free surface in the laboratory basin. In a fluid of uniform density, free surface variations provide a source of relative vorticity through the mechanism of vortex stretching or compression (e.g., Pedlosky, 1987). In a two-layer fluid, however, such variations are proportionally less, by a factor of $\Delta \rho / \rho$, than the deformation of the interface between the two layers. Hence, to order $\Delta \rho / \rho$, the motion of the free surface is negligible compared to the motion of the interface and the free surface appears rigid, insofar as the vertical velocity is concerned (e.g., Pedlosky, 1987). Consequently, free surface variations are unlikely to be dynamically significant in our experiments. It can be shown that a radial variation of the free surface caused by background rotation, $\bar{\eta}$, does not modify the governing equation for $h$ (when written in cylindrical coordinates) and the expressions for $\kappa_{s}, \kappa_{a}(23 \mathrm{a}-$ 23b), provided that the layer depth in these expressions is identified as $h+\bar{\eta}$.

\section{b. Effect of mixing}

The reduced gravity which is assumed in our numerical solutions is based on density measurements on freshwater and seawater samples which were made prior to the laboratory experiments. It has therefore a constant value. In the laboratory experiments, on the other hand, the density of the buoyant layer is expected to change, at least to some extent, due to mixing with ambient seawater. Sampling the buoyant layer during experiments such as reported here, however, is difficult given the small layer thickness (of the order of $1 \mathrm{~mm}$ ) and the relatively high rotation rate $\left(\Omega=0.5 \mathrm{~s}^{-1}\right)$. Accordingly, the density of the buoyant layer was not measured and the amount of mixing which may have occurred during our experiments is unknown.

On the other hand, it is unclear that turbulent mixing played a significant role in our experiments. First, turbulence was not noticeable, except perhaps within the bulge. There irregular and intermittent streaks of very red water were observed, which may have been produced by the upward motion of buoyant water bursting from the sponge. Second, it seems unlikely that mixing is the sole explanation for the differences between the experimental results and the numerical solutions. To illustrate this point, the equation for $h(22)$ is again solved numerically for each experimental value of $q$ but with the reduced gravity arbitrarily set to $g^{\prime}=0.12 \mathrm{~m} \mathrm{~s}^{-2}$, which is half the measured value. The kinematical viscosity and the Coriolis parameter are unchanged. Whereas the distance of the salinity front from the wall predicted with $g^{\prime}=0.12 \mathrm{~m} \mathrm{~s}^{-2}$ agrees better with observations for small values of $q$, the agreement is clearly worse for high $q$ (Figs. 11a-11f). A smaller value of $g^{\prime}$ reduces the mean speed of the salinity front propagation away from the wall (Figs. 11a-11f), which 
would worsen the agreement with the observed speeds (Fig. 12). Thus, the occurrence of mixing in our experiments is unlikely to universally explain the discrepancies between the experimental and numerical results.

\section{c. Effect of surface tension}

A possible effect of surface tension on gravity currents produced in the laboratory has been mentioned in earlier work (McClimans and Saegrov, 1982). Consider the triple contact where the front between two liquid layers (liquid 1 above liquid 2) intersects the air, i.e., the free surface. The general condition for the upper liquid to initially spread on the lower liquid due to surface tension is (Davis and Rideal, 1963)

$$
\gamma_{2 a}>\gamma_{1 a}+\gamma_{12}
$$

Here $\gamma_{1 a}\left(\gamma_{2 a}\right)$ is the surface tension of liquid 1 (2) against air and $\gamma_{12}$ is the surface tension between the two liquids. The difference $\gamma_{2 a}-\left(\gamma_{1 a}+\gamma_{12}\right)$ is called the spreading coefficient. In (36) the surface tensions are those before mutual saturation of the liquids has occurred and where, if the liquids are ultimately miscible, $\gamma_{12}$ vanishes (Davis and Rideal, 1963). Thus, if the liquids are freshwater and seawater (miscible), condition (36) reduces to

$$
\gamma_{2 a}>\gamma_{1 a}
$$

The surface tension of seawater against air is slightly greater (by $\mathrm{O}\left(10^{-3}\right) \mathrm{N} \mathrm{m}^{-1}$ ) than that of pure water (Neumann and Pierson, 1966), suggesting that the above condition is met in our experiments: at least initially, freshwater would spread on seawater. Whether this effect is quantitatively important in our experiments, however, is difficult to establish given the inevitable presence of surface impurities. Such impurities may greatly lower surface tensions and usually reduce the spreading coefficient (Davis and Rideal, 1963).

The consideration of a surface tension gradient in the theory has some remarkable consequences (Appendix D). For example, such a gradient produces the divergence (or convergence) of an additional flux of volume in the upper layer, which tends to decrease (or increase) the layer depth. The additional flux divergence tends to advect the front in the direction of increasing surface tension, i.e., perpendicularly to the front line. These results support the notion that a surface tension gradient tends to pull buoyant plumes outwards in laboratory experiments (McClimans and Saegrov, 1982). On the other hand, their validity is probably limited as surface tension in a two-layer theory may be best regarded as discontinuous at the front between two layers. Further analysis of this interesting problem is beyond the scope of this paper.

\section{d. Other possible effects}

Other effects might contribute to the discrepancies between our experimental and numerical results. One such effect is the formation of a recirculating bulge near the freshwater source. A bulge was observed to grow in size in our laboratory experiments, although no 
specific measurements on the bulge were taken. The bulge is an inertial effect due to potential vorticity conservation in a plume of shrinking thickness near the source (e.g., Yankovsky and Chapman, 1997; Nof and Pichevin, 2001). The resulting anticyclonic vortex near the source competes with Kelvin wave dynamics, as in the related problem of nonlinear Rossby adjustment in a channel (Hermann et al., 1989).

Earlier laboratory experiments with a rotating basin have determined that a significant fraction of the buoyant water exiting the source may be stored in a bulge near the source (Avicola and Huq, 2003b; Horner-Devine et al., 2006). Consequently, the length and width of the gravity current along the wall are much reduced if a bulge is present near the source (Avicola and Huq, 2003b). The numerical solutions which are considered in this paper do not reproduce a bulge, likely because of the omission of inertia in the theory (e.g., Yankovsky and Chapman, 1997; Nof and Pichevin, 2001). The lack of a bulge might explain why some of these solutions overestimate the width of gravity current along the wall compared to observations for the smallest values of $q$ (Figs. 11a-11b). It is less clear, on the other hand, why the speed of the salinity front migration away from the wall would then be generally underestimated (Fig. 12).

Another possible effect is horizontal friction, in particular along the wall and along the salinity front. The presence of horizontal boundary layers would invalidate the scaling arguments of Section 3, since the horizontal scale of variation of dynamical variables such as $u, v$ would be much lower in these layers than elsewhere. Consequently, the horizontal friction terms in $(4 a-4 b)$ would not be negligible compared to the Coriolis acceleration in these layers. Speculatively, horizontal friction would retard the movement of buoyant water along the wall and hence contribute to the overestimation of the distance of the salinity front from the wall in some numerical solutions (Figs. 11a-11b). On the other hand, it is again less clear how horizontal friction could account for the fact that the speed at which the front moves away from the wall is generally underestimated (Fig. 12).

\section{Summary}

Simple experiments are conducted in the laboratory, whereby the movement of buoyant water released in a sectorial basin inscribed in a cylindrical tank is investigated. The basin has an angle of $120^{\circ}$ at the apex, a radius of $1 \mathrm{~m}$, vertical walls, and a flat bottom. Rotation is anticlockwise and proceeds at the same rate in all experiments $\left(f=1 \mathrm{~s}^{-1}\right)$. The wall along which freshwater is released runs from the apex to the arc of the basin and is seen to be on the left from the arc. It has a corner with a right angle on the inner side. The basin initially contains salt water that is brought to solid body rotation. Freshwater is then released near the surface along the wall between the apex and the corner. The rate of freshwater inflow is kept constant in a given experiment and varies between experiments from about $1 \mathrm{ml} \mathrm{s}^{-1}$ to about $4.5 \mathrm{ml} \mathrm{s}^{-1}$.

The freshwater forms a bulge near the source and a gravity current then flows toward the corner $\mathrm{C} 1$ along the wall. The current does not separate at the corner and remains attached 
to the wall. Depending on the experiments, the along-wall velocity $U_{\|}$between the source and corner $\mathrm{C} 1$ ranges from $3 \mathrm{~mm} \mathrm{~s}^{-1}$ to $30 \mathrm{~mm} \mathrm{~s}^{-1}$, implying a critical radius of curvature $U_{\|} / f$ of $3 \mathrm{~mm}$ to $30 \mathrm{~mm}$ (Boormans and Garrett, 1989). Although the radius of curvature of corner $\mathrm{C} 1$, which is very sharp, has not been measured, it is clearly smaller than $30 \mathrm{~mm}$. Consequently, the gravity current should have detached from the wall at the corner in some experiments according to the criterion of Boormans and Garrett (1989). We speculate that the lack of detachment stems from the small rates of freshwater inflow which have been considered in our experiments, leading to gravity currents that are dynamically different from those simulated in previous laboratory studies and envisioned in inviscid theories of current separation from a curved boundary (e.g., Klinger, 1994). In other words, the lack of detachment in our experiments might be due to the low Reynolds numbers that characterize these experiments.

The salinity front along the wall migrates away from the wall with a time scale greatly exceeding the rotation period. The front reaches a distance of several deformation radii $\left(2.6 \mathrm{~cm} \leq l_{D} \leq 3.8 \mathrm{~cm}\right)$ over the course of the experiments (from about 5 to 15 minutes). The migration of the front can be described to a good approximation as a motion with constant deceleration. The migration speed near the wall and the deceleration rate of the front both increase with the rate of freshwater inflow.

The migration of the salinity front away from the wall is down the pressure gradient, indicating that the geostrophic constraint that tends to maintain buoyant water along the wall is eventually broken. A simple theory of a surface layer containing light fluid spreading over an infinitely deep layer containing dense fluid is considered to explore the role of vertical friction in the migration of the front observed in the laboratory. In the upper layer, horizontal momentum is driven by a balance between Coriolis acceleration, horizontal pressure gradient, and vertical friction. In the lower layer, the horizontal pressure gradient is ignored. In both layers vertical friction takes the form $v \partial^{2}(u, v) / \partial z^{2}$.

Analysis of the equations of motion shows that the depth of the buoyant layer $(h)$ satisfies a diffusion equation,

$$
\frac{\partial h}{\partial t}=\nabla \cdot(\mathbf{K}(h) \nabla h) .
$$

The diffusion tensor $\mathbf{K}$ is the sum of a symmetric part representing the effect of vertical friction and an antisymmetric part representing the effect of geostrophic motion. Note that this equation can be transformed to an advection-diffusion equation.

Previous examples of diffusion or advection-diffusion equations for layer thickness can be found in geophysical fluids dynamics. For instance, the layers of homogeneous temperature and salinity which are observed at some oceanic locations from microstructure data could spread under the action of vertical friction at the bottom and top of the layers. The spreading was described by a diffusion equation, with an effective diffusivity given by $g^{\prime} \sqrt{\nu / f^{3}}$ (Stommel and Fedorov, 1967). This is four times greater than $\kappa_{0}$, which is the diffusivity obtained in the limit $h / \delta \gg 1$ and implicit in (31). The depth average of a scalar property in a 
bounded shear flow is governed by an advection-diffusion equation where the components of the diffusion tensor depend on the departures of the velocity components from their average values (Fischer, 1978). The surface elevation of a thin layer of homogeneous laminar fluid subject to rotation and bottom friction obeys to a diffusion equation for horizontal scales larger than the Rossby radius, where the effective diffusivity is $g \sqrt{\nu /\left(2 f^{3}\right)}$ (Gill, 1982). The effective diffusivity is reduced to $g^{\prime} \sqrt{\nu /\left(2 f^{3}\right)}$ if the layer is sandwiched between an infinitely thick layer above and the bottom below (Gill et al., 1979). An homogeneous intrusion in a rotating stratified fluid (Gill, 1981) and a buoyant layer floating above a denser, rotating layer (e.g. Garrett and Loder, 1981; Wright, 1989) each have a diffusive behavior, with an effective diffusivity of magnitude $v(N / f)^{2}$, where $N$ is the buoyancy frequency. In the boundary layer along the slope of a rotating and stratified fluid, the velocity component along the slope 'diffuses' away from the slope with an effective diffusivity depending on $\nu, N / f$, the slope angle, and the Prandtl number (MacCready and Rhines, 1991). Our simple theory extends all these earlier studies by considering the evolution of a density front between two layers of different densities in concert with a representation of vertical friction that is precise for laboratory experiments.

Numerical solutions of the governing equation of $h$ for arbitrary $h / \delta$ are compared to observations made in the laboratory. The initial conditions, boundary conditions, and parameters of the equation are all constrained from laboratory conditions, providing a stringent test. Comparison of numerical solutions with observations is generally favourable. The gradual widening of the gravity current and its uninterrupted contact with the wall (no separation at corner $\mathrm{C} 1$ ) are reproduced in the solutions.

The movement of the salinity front observed in the laboratory (Figs. 4-6) could be understood from the following scaling argument. Consider two basic observations (Fig. 10a). First, the front is about parallel to the wall, suggesting that $\partial h / \partial y \ll \partial h / \partial x$ is a reasonable approximation, at least at some locations. Second, the buoyant layer is deeper near the wall than away from the wall as revealed by the gradual color change in the cross-front direction, suggesting that $\partial h / \partial x<0$. Both observations suggests that the evolution of the buoyant layer is approximately governed by $\partial h / \partial t=(\partial / \partial x)\left(\kappa_{s} \partial h / \partial x\right)=$ $\left(\partial \kappa_{s} / \partial x\right)(\partial h / \partial x)+\kappa_{s}\left(\partial^{2} h / \partial x^{2}\right)$ where $\partial \kappa_{s} / \partial x$ is negative since $\partial h / \partial x$ is negative (in our experiments $h$ is of the order of $\delta$ or less, so $\kappa_{s}$ generally increases with $h$; Fig. 9). At the front, $\kappa_{s}$ vanishes since $h$ is zero, so the rate of change of $h$ at the front is governed by $\partial h / \partial t+\left(-\partial \kappa_{s} / \partial x\right)(\partial h / \partial x)=0$, where the effective velocity $\left(-\partial \kappa_{s} / \partial x\right)$ arises from vertical friction at the interface between the two layers. As the front is near the wall, the depth of the buoyant layer changes from its maximum value at the wall $\left(h_{w}\right.$, which is fixed by experimental conditions) to zero at the front over a relatively small distance. Accordingly, the scale for $\left(-\partial \kappa_{s} / \partial x\right)$ is large near the wall and the front moves quickly. In contrast, away from the wall, the change in layer depth is less over the same distance, the scale for $\left(-\partial \kappa_{s} / \partial x\right)$ is small, and the front moves slowly. Thus, the front decelerates as it moves away from the wall (Fig. 4). This argument would also explain why the speed of the front near the wall increases with the rate of freshwater inflow (Fig. 5), since $h_{w}$ and hence the scale for 
$\left(-\partial \kappa_{s} / \partial x\right)$ near the wall increase with $q$. It would also account for the front deceleration increasing in amplitude with $q$ (Fig. 6), since the change in the effective velocity $\left(-\partial \kappa_{s} / \partial x\right)$ between a location near the wall and a location away from the wall is characterized by a larger value as $h_{w}$ is larger.

On the other hand, differences between numerical solutions and observations exist that are not fully understood. A gradient in surface tension across the salinity front, the formation of an inertial bulge near the freshwater inlet, and horizontal friction may each contribute to the differences.

To directly apply our results to the real ocean requires a leap that we are not prepared to attempt given the idealized character of the laboratory experiments and the simplicity of the theory. Spreading of density fronts in the ocean is likely to be dominated by processes, such as frontal instabilities and mixing by eddies, which are not considered in this paper. Turbulent mixing appears as an important process for the dynamics of buoyant coastal plumes, as indicated by field observations (e.g., Münchow and Garvine, 1993; Sanders and Garvine, 2001; MacDonald and Geyer, 2004; Pritchard and Huntley, 2006; Horner-Devine et al., 2009) and numerical simulations with circulation models (e.g., Fong and Geyer, 2001; Hetland, 2005; Pimenta et al., 2011). Nonetheless, the notion that interfacial friction leads to seaward spreading of density fronts bounding buoyant water introduced along oceanic boundaries (e.g., Wright, 1989) finds here apparent support.

Acknowledgments. We thank two anonymous reviewers for a very careful reading of this manuscript and for constructive comments that allowed us to improve it significantly. The help of Claudia Cenedese in the laboratory is gratefully acknowledged. Rocky Geyer pointed to us recent studies on river plumes in the ocean. This study was supported by the Ocean and Climate Change Institute at WHOI.

\section{APPENDIX A}

\section{Details about laboratory experiments}

Set-up

The rotating table contains several elements enabling the release of freshwater into the basin during rotation (Fig. 1). A pail filled with fresh (tap) water is fixed outside the sectorial basin. A red dye (food color) is added in constant proportion to the freshwater, so the evolution of freshwater in the basin could be observed. The freshwater is pumped from the pail into the basin using a pump that is fastened on a shelf outside the basin. The freshwater from the pail flows through a tube (via the pump) toward the wall. The tube is connected to a block of PVC that is attached to the outer side of the wall. The block has a large threaded entry hole and five exit holes. A PVC fitting that fits the tube is fixed in the threading. The exit holes are facing a sponge that is embedded in a square opening made in the wall. The sponge is intended to distribute the freshwater over the area of the opening before freshwater flows into the basin. The sponge is inserted into the opening in such a way that it protrudes 
from the wall by only a small distance. The sides of the opening have a length of $5 \mathrm{~cm}$. The opening is straight with vertical sides located at a distance of $10 \mathrm{~cm}$ and $15 \mathrm{~cm}$ from the apex of the basin. Its lower side is at a height of slightly less than $18 \mathrm{~cm}$ above the bottom of the basin.

Both the sectorial basin and the other, passive basin are filled with seawater to reduce the pressure difference on each side of the basin walls. The level of seawater above the bottom in these basins is typically $0.23 \pm 0.01 \mathrm{~m}$. The sectorial basin is entirely covered by a lid in order to reduce the action of a wind stress on the water surface during rotation (Fig. 2). The lid is transparent, made of polycarbonate, and has a thickness of about $2 \mathrm{~mm}$ (light refraction at the lower and upper surfaces of the lid is negligible). It lies on the vertical walls of the basin and is clamped at two places along the periphery of the table. The space between the lower edge of the lid and the wall that is visible in some places is obstructed with plastic bands adhering electrostatically and glued with gummed paper along the basin to prevent air exchange.

\section{Spin-up}

The time required to spin up a fluid of uniform density by rotation communicated through a bottom Ekman layer is $H / \sqrt{2 v f}$, where $H$ is the depth of the fluid, $v$ the kinematic viscosity, and $f$ the Coriolis parameter (e.g., Pedlosky, 1987). For $H=0.23 \mathrm{~m}, v=10^{-6} \mathrm{~m}^{2} \mathrm{~s}^{-1}$, and $f=1 \mathrm{~s}^{-1}$, the spin-up time amounts to $163 \mathrm{~s}$. For all our experiments, a time of at least 30 minutes, which corresponds to more than ten spin-up times, elapses between the start of rotation and the start of freshwater discharge. Accordingly, the salt water in the basin should always be close to a state of solid body rotation when the discharge is initiated.

Note that, owing to rotation, the free surface in the basin becomes parabolic. Let $r$ be an arbitrary radial distance from the apex and $R$ the basin radius. Under the effect of rotation, the level of salt water in our experiments drops where $r<R / \sqrt{2}$ and rises where $r>R / \sqrt{2}$, with $R / \sqrt{2}=71 \mathrm{~cm}$. At radial distances $10 \mathrm{~cm}<r<15 \mathrm{~cm}$, where the sponge is positioned, the level is depressed by $6 \mathrm{~mm}$. These considerations dictate that the sectorial basin be filled with salt water to a level equal to the height of the upper side of the sponge above the bottom plus at least $6 \mathrm{~mm}$ in order for the sponge to remain submerged during rotation. For all our experiments, the upper side of the sponge is below the water surface by a few millimeters prior to rotation and most of the sponge remains submerged during rotation. Thus, freshwater is always introduced near the water surface during the experiments.

\section{Movement of salinity front}

For each experiment, the movement of the salinity front is determined by measuring the time it takes for the front to reach ten different apparent distances from the wall (from $l_{\mathrm{o}}=2 \mathrm{~cm}$ to $l_{\mathrm{o}}=38 \mathrm{~cm}$, with a spacing of $4 \mathrm{~cm}$ ). Each distance is identified on the 
digitized sequence by a straight line which is parallel to the wall and which passes through the appropriate marking on top of the adjacent wall. For each distance two values are determined from the sequence: the time when the front is last seen on the wall side of the line and the time when the front is first seen on the other side of the line. The mean of the two values is taken as an approximate estimate of the time it takes for the front to reach a particular distance from the wall, and the range of the two values is taken as a conservative estimate of the uncertainty in this time.

\section{APPENDIX B}

\section{Derivation of the equation for $h$}

The depth integral of the velocity component $u_{1}$,

$$
\int_{-h}^{0} u_{1} d z=A_{1} \int_{-h}^{0}\left(\cos \frac{z}{\delta} \cosh \frac{z}{\delta}\right) d z-B_{1} \int_{-h}^{0}\left(\sin \frac{z}{\delta} \sinh \frac{z}{\delta}\right) d z+h u_{g}
$$

is evaluated by integration by parts, which gives

$$
\begin{aligned}
\int_{-h}^{0} u_{1} d z= & A_{1} \frac{\delta}{2}\left(\cos \frac{h}{\delta} \sinh \frac{h}{\delta}+\sin \frac{h}{\delta} \cosh \frac{h}{\delta}\right) \\
& -B_{1} \frac{\delta}{2}\left(\sin \frac{h}{\delta} \cosh \frac{h}{\delta}-\cos \frac{h}{\delta} \sinh \frac{h}{\delta}\right)+h u_{g} .
\end{aligned}
$$

Equivalently,

$$
\int_{-h}^{0} u_{1} d z=\frac{\delta}{2}\left\{\left(A_{1}+B_{1}\right) \cos \frac{h}{\delta} \sinh \frac{h}{\delta}+\left(A_{1}-B_{1}\right) \sin \frac{h}{\delta} \cosh \frac{h}{\delta}\right\}+h u_{g},
$$

where

$$
\begin{aligned}
& A_{1}+B_{1}=e^{-h / \delta}\left\{u_{g}\left(\sin \frac{h}{\delta}-\cos \frac{h}{\delta}\right)-v_{g}\left(\sin \frac{h}{\delta}+\cos \frac{h}{\delta}\right)\right\}, \\
& A_{1}-B_{1}=e^{-h / \delta}\left\{v_{g}\left(\cos \frac{h}{\delta}-\sin \frac{h}{\delta}\right)-u_{g}\left(\sin \frac{h}{\delta}+\cos \frac{h}{\delta}\right)\right\} .
\end{aligned}
$$


Inserting (B4a-B4b) into (B3) yields

$$
\begin{aligned}
\int_{-h}^{0} u_{1} d z= & \frac{\delta}{2} e^{-h / \delta} u_{g}\left\{-\cos ^{2} \frac{h}{\delta} \sinh \frac{h}{\delta}+\cos \frac{h}{\delta} \sin \frac{h}{\delta}\left(\sinh \frac{h}{\delta}-\cosh \frac{h}{\delta}\right)\right. \\
& \left.-\sin ^{2} \frac{h}{\delta} \cosh \frac{h}{\delta}\right\} \\
& +\frac{\delta}{2} e^{-h / \delta} v_{g}\left\{-\cos ^{2} \frac{h}{\delta} \sinh \frac{h}{\delta}+\cos \frac{h}{\delta} \sin \frac{h}{\delta}\left(\cosh \frac{h}{\delta}-\sinh \frac{h}{\delta}\right)\right. \\
& \left.-\sin ^{2} \frac{h}{\delta} \cosh \frac{h}{\delta}\right\}+h u_{g} .
\end{aligned}
$$

Expressing the hyperbolic functions in terms of exponentials and using formulas for circular functions, this integral becomes

$$
\begin{aligned}
\int_{-h}^{0} u_{1} d z= & \frac{\delta}{8} u_{g}\left\{\left(1+\cos \frac{2 h}{\delta}\right)\left(e^{-2 h / \delta}-1\right)-2 \sin \frac{2 h}{\delta} e^{-2 h / \delta}\right. \\
& \left.-\left(1-\cos \frac{2 h}{\delta}\right)\left(1+e^{-2 h / \delta}\right)\right\} \\
& +\frac{\delta}{8} v_{g}\left\{\left(1+\cos \frac{2 h}{\delta}\right)\left(e^{-2 h / \delta}-1\right)+2 \sin \frac{2 h}{\delta} e^{-2 h / \delta}\right. \\
& \left.-\left(1-\cos \frac{2 h}{\delta}\right)\left(1+e^{-2 h / \delta}\right)\right\}+h u_{g} .
\end{aligned}
$$

Distributing the terms between parentheses and since $\left(u_{g}, v_{g}\right)=\left(g^{\prime} / f\right)(-\partial h / \partial y, \partial h / \partial x)$, the integral can finally be written as

$$
\begin{aligned}
\int_{-h}^{0} u_{1} d z= & -\frac{g^{\prime} \delta}{4 f}\left\{1-e^{-2 h / \delta}\left(\sin \frac{2 h}{\delta}+\cos \frac{2 h}{\delta}\right)\right\} \frac{\partial h}{\partial x} \\
& +\frac{g^{\prime} \delta}{4 f}\left\{1+e^{-2 h / \delta}\left(\sin \frac{2 h}{\delta}-\cos \frac{2 h}{\delta}\right)-4 \frac{h}{\delta}\right\} \frac{\partial h}{\partial y},
\end{aligned}
$$

where the quantities multiplying $\partial h / \partial x, \partial h / \partial y$ are minus the effective diffusivities $\kappa_{s}, \kappa_{a}$ (23a-23b). The depth integral of the velocity component $v_{1}$ is calculated similarly. Inserting the depth integrals of $u_{1}, v_{1}$ into the statement of volume conservation (13c) leads to the governing equation (22). 


\section{APPENDIX C}

\section{Numerical method of solution}

The equation for the layer depth $h$ (34) is discretized on a regular grid with spacing $\Delta x=\Delta y=1 \mathrm{~cm}$ between grid points. The layer depth $h$ is defined at grid points, and the effective velocities $\bar{u}$ and $\bar{v}$ are defined midway between grid points in the directions $x$ and $y$, respectively. Grid points adjacent to a boundary are at a distance $\Delta x / 2$ and/or $\Delta y / 2$ from the boundary. Below, the indexes $i$ and $j$ refer to discrete values of the horizontal coordinates $x$ and $y$, respectively, and the index $n$ refers to discrete values of time. The overbar for the effectives velocities is omitted for clarity.

The method of solution is a finite-difference algorithm called MPDATA (see Smolarkiewicz and Margolin (1998); and references therein). This method is iterative. The first pass is a simple donor cell approximation,

$$
h_{i, j}^{*}=h_{i, j}^{n}-\left[(u h)_{i+1 / 2, j}-(u h)_{i-1 / 2, j}\right] \frac{\Delta t}{\Delta x}-\left[(v h)_{i, j+1 / 2}-(v h)_{i, j-1 / 2}\right] \frac{\Delta t}{\Delta y},
$$

where $\Delta t$ is the time step. The flux $(u h)_{i+1 / 2, j}$, for example, is computed from

$$
(u h)_{i+1 / 2, j}=0.5\left[\left(u_{i+1 / 2, j}+\left|u_{i+1 / 2, j}\right|\right) h_{i, j}^{n}+\left(u_{i+1 / 2, j}-\left|u_{i+1 / 2, j}\right|\right) h_{i+1, j}^{n}\right] .
$$

Here $u_{i+1 / 2, j}$ is a time-intermediate value evaluated with second-order accuracy (Smolarkiewicz and Clark, 1986),

$$
u_{i+1 / 2, j}=0.5\left(3 u_{i+1 / 2, j}^{n}-u_{i+1 / 2, j}^{n-1}\right) .
$$

The velocity component $u_{i+1 / 2, j}^{n}$ is given by $u_{i+1 / 2, j}^{n}=u_{i+1 / 2, j}^{n, d}+u_{i+1 / 2, j}^{n, a}$, where

$$
\begin{aligned}
& u_{i+1 / 2, j}^{n, d}=\frac{-1}{\left(\sum h\right)_{d}}\left[\kappa_{s}\left(h_{i, j}^{n}\right) \frac{h_{i+1, j}^{n}-h_{i, j}^{n}}{\Delta x}+\kappa_{s}\left(h_{i+1, j}^{n}\right) \frac{h_{i+1, j}^{n}-h_{i, j}^{n}}{\Delta x}\right], \\
& u_{i+1 / 2, j}^{n, a}=\frac{-1}{\left(\sum h\right)_{a}}\left[\kappa_{a}\left(h_{i, j}^{n}\right) \frac{h_{i, j+1}^{n}-h_{i, j-1}^{n}}{2 \Delta y}+\kappa_{a}\left(h_{i+1, j}^{n}\right) \frac{h_{i+1, j+1}^{n}-h_{i+1, j-1}^{n}}{2 \Delta y}\right]
\end{aligned}
$$

with

$$
\begin{aligned}
& \left(\sum h\right)_{d}=h_{i+1, j}^{n}+h_{i, j}^{n}+\epsilon, \\
& \left(\sum h\right)_{a}=0.5\left(h_{i, j+1}^{n}+h_{i, j-1}^{n}+h_{i+1, j+1}^{n}+h_{i+1, j-1}^{n}\right)+\epsilon .
\end{aligned}
$$

The quantity $\epsilon$ is a small value $\left(10^{-15}\right)$ to ensure $u_{i+1 / 2, j}^{n, d}=0$ when $h_{i+1, j}^{n}=h_{i, j}^{n}=0$ and $u_{i+1 / 2, j}^{n, a}=0$ when $h_{i, j+1}^{n}=h_{i, j-1}^{n}=h_{i+1, j+1}^{n}=h_{i+1, j-1}^{n}=0$. The effective diffusivities $\kappa_{s}$ and $\kappa_{a}$ are computed from layer depth $h$ using equations (23a) and (23b), respectively. Note that the evaluation of $u_{i+1 / 2, j}^{n, a}$ relies on the value of $h$ at the four neighboring points $(i, j-1),(i+1, j-1),(i, j+1)$, and $(i+1, j+1)$. If one or several of these points 
is outside the domain, then $u_{i+1 / 2, j}^{n, a}$ is evaluated from one-sided difference schemes with second-order accuracy. Expressions similar to (C2-C5a) are used to evalue the three other fluxes in the donor cell approximation (C1).

The second pass of MPDATA increases the accuracy by estimating and compensating the (second-order) truncation error of the first pass,

$$
h_{i, j}^{n+1}=h_{i, j}^{*}-\left[(\tilde{u} h)_{i+1 / 2, j}-(\tilde{u} h)_{i-1 / 2, j}\right] \frac{\Delta t}{\Delta x}-\left[(\tilde{v} h)_{i, j+1 / 2}-(\tilde{v} h)_{i, j-1 / 2}\right] \frac{\Delta t}{\Delta y} .
$$

The flux $(\tilde{u} h)_{i+1 / 2, j}$, for example, is calculated as

$$
(\tilde{u} h)_{i+1 / 2, j}=0.5\left[\left(\tilde{u}_{i+1 / 2, j}+\left|\tilde{u}_{i+1 / 2, j}\right|\right) h_{i, j}^{n}+\left(\tilde{u}_{i+1 / 2, j}-\left|\tilde{u}_{i+1 / 2, j}\right|\right) h_{i+1, j}^{n}\right],
$$

where $\tilde{u}_{i+1 / 2, j}$ is an 'antidiffusive' velocity. The antidiffusive velocity $\tilde{u}_{i+1 / 2, j}$ is given by $\tilde{u}_{i+1 / 2, j}=\tilde{u}_{i+1 / 2, j}^{(1)}+\tilde{u}_{i+1 / 2, j}^{(2)}$. where

$$
\begin{aligned}
& \tilde{u}_{i+1 / 2, j}^{(1)}=\left(\left|u_{i+1 / 2, j}\right| \Delta x-u_{i+1 / 2, j}^{2} \Delta t\right) \frac{h_{i+1, j}^{*}-h_{i, j}^{*}}{\left(h_{i+1, j}^{*}+h_{i, j}^{*}+\epsilon\right) \Delta x}, \\
& \tilde{u}_{i+1 / 2, j}^{(2)}=-0.125 \Delta t u_{i+1 / 2, j}\left\langle v_{i+1 / 2, j}\right\rangle \frac{h_{i, j+1}^{*}-h_{i, j-1}^{*}+h_{i+1, j+1}^{*}-h_{i+1, j-1}^{*}}{\left(\sum h\right) \Delta y},
\end{aligned}
$$

with

$$
\begin{aligned}
\left\langle v_{i+1 / 2, j}\right\rangle & =0.25\left(v_{i, j-1 / 2}+v_{i+1, j-1 / 2}+v_{i+1, j+1 / 2}+v_{i, j+1 / 2}\right), \\
\sum h & =0.25\left(h_{i, j+1}^{*}+h_{i, j-1}^{*}+h_{i+1, j+1}^{*}+h_{i+1, j-1}^{*}\right)+\epsilon .
\end{aligned}
$$

The second contribution $\tilde{u}_{i+1 / 2, j}^{(2)}$ corrects for the presence of a cross derivative that appears for advection problems with more than one spatial dimension. It also relies on the value of layer depth $h$ at the four neighboring points $(i, j-1),(i+1, j-1),(i, j+1)$, and $(i+1, j+1)$. If one or several of these points is outside the domain, then $\tilde{u}_{i+1 / 2, j}^{(2)}$ is also evaluated from one-sided difference schemes with second-order accuracy.

\section{APPENDIX D}

\section{Effect of surface tension}

Generally, a variation in surface tension along the surface of separation between a liquid and a gas gives rise to a tangential stress on this surface (e.g., Landau and Lifshitz, 1959; Levich and Krylov, 1969). This stress induces convective motion in at least one of the fluids 
on each side of the surface - the Marangoni effect. In order to incorporate this stress in our theory, the surface conditions (14) is replaced by (e.g., Levich and Krylov, 1969)

$$
\begin{aligned}
& \rho_{1} \nu\left(\frac{\partial w_{1}}{\partial x}+\frac{\partial u_{1}}{\partial z}\right)=\frac{\partial \gamma}{\partial x}, \\
& \rho_{1} \nu\left(\frac{\partial w_{1}}{\partial y}+\frac{\partial v_{1}}{\partial z}\right)=\frac{\partial \gamma}{\partial y},
\end{aligned}
$$

where the relative motion of the air above the water has been neglected compared to that of the water. If the free surface is flat $\left(w_{1}=0\right)$, these conditions reduce to

$$
\rho_{1} \nu \frac{\partial u_{1}}{\partial z}=\frac{\partial \gamma}{\partial x} \quad \text { and } \quad \rho_{1} \nu \frac{\partial v_{1}}{\partial z}=\frac{\partial \gamma}{\partial y} .
$$

Several consequences of the altered conditions (D2) are worth noting. First, the components of horizontal velocity in the upper layer (15a) and (15b) are augmented to include a contribution equal to, respectively,

$$
\begin{aligned}
& u_{\gamma}=e^{-z / \delta}\left\{u_{\gamma *}\left(\cos \frac{h}{\delta}+\sin \frac{h}{\delta}\right)+v_{\gamma *}\left(\cos \frac{h}{\delta}-\sin \frac{h}{\delta}\right)\right\}, \\
& v_{\gamma}=e^{-z / \delta}\left\{u_{\gamma *}\left(\sin \frac{h}{\delta}-\cos \frac{h}{\delta}\right)+v_{\gamma *}\left(\sin \frac{h}{\delta}+\cos \frac{h}{\delta}\right)\right\},
\end{aligned}
$$

where $u_{\gamma *}, v_{\gamma *}=\left(\delta / 2 \rho_{1} \nu\right)(\partial \gamma / \partial x, \partial \gamma / \partial y)$. The constants $A_{1}, B_{1}(20 \mathrm{a}-20 \mathrm{~b})$ are unchanged. In contrast, the constants $A_{2}$ and $B_{2}$, which are given by (21a) and (21b), are augmented to include, respectively, the sum $u_{\gamma^{*}}+v_{\gamma *}$ and the difference $v_{\gamma *}-u_{\gamma^{*}}$.

Accordingly, a gradient in surface tension produces a different effect in the two layers. In the upper layer, an Ekman spiral (defined by D3a-D3b) is produced near the surface in addition to the spiral caused by vertical friction at the layer interface. The spiral caused by vertical friction at the layer interface is not modified by $\nabla \gamma$. In contrast, in the deep layer, no new spiral is produced but the spiral due to vertical friction at the layer interface is modified by $\nabla \gamma$. Note that, in both layers, the contribution of the surface tension gradient to the horizontal velocities decays with depth with the Ekman layer scale $\delta$.

A second consequence of the altered conditions (D2) is the addition of a term in the governing equation for the depth of the buoyant layer,

$$
\frac{\partial h}{\partial t}+\nabla \cdot\left(\mathbf{u}_{\gamma *} \delta\right)=\nabla \cdot(\mathbf{K}(h) \nabla h),
$$

where the components of the effective velocity $\mathbf{u}_{\gamma *}$ are

$$
\begin{aligned}
& \hat{x} \cdot \mathbf{u}_{\gamma *}=u_{\gamma *} e^{-h / \delta} \sin \frac{h}{\delta}+v_{\gamma *}\left(1-e^{-h / \delta} \cos \frac{h}{\delta}\right), \\
& \hat{y} \cdot \mathbf{u}_{\gamma *}=v_{\gamma *} e^{-h / \delta} \sin \frac{h}{\delta}-u_{\gamma *}\left(1-e^{-h / \delta} \cos \frac{h}{\delta}\right) .
\end{aligned}
$$


The diffusion tensor $\mathbf{K}$ is not modified by $\nabla \gamma$ and is still given by (25). Hence, the surface tension gradient leads to the divergence (or convergence) of an additional flux of volume in the upper layer. A divergence of volume flux, for example, tends to decrease the layer depth. This effect is analogous to the Ekman suction caused by the divergence of wind-driven currents near the ocean surface.

At the front (where $h=0$ ), the additional flux divergence due to the surface tension gradient reduces to

$$
\nabla \cdot\left(\mathbf{u}_{\gamma *} \delta\right)=\left(u_{\gamma *}+v_{\gamma *}\right) \frac{\partial h}{\partial x}+\left(v_{\gamma *}-u_{\gamma *}\right) \frac{\partial h}{\partial y} .
$$

If the $y$ axis, for example, is aligned with the front, so that both $\partial h / \partial y$ and $\partial \gamma / \partial y$ vanish, this term becomes

$$
\nabla \cdot\left(\mathbf{u}_{\gamma *} \delta\right)=u_{\gamma *} \frac{\partial h}{\partial x} .
$$

In this case, the front tends to be advected perpendicularly to the front line, in the direction of increasing surface tension, and at the speed $u_{\gamma *}=\left(\delta / 2 \rho_{1} \nu\right) \partial \gamma / \partial x$. A similar result is obtained if the $x$ axis is aligned with the front.

\section{REFERENCES}

Avicola, G. and P. Huq. 2002. Scaling analysis for the interaction between a buoyant coastal current and the continental shelf: Experiment and observations. J. Phys. Oceanogr., 32, 3233-3248.

2003a. The role of outflow geometry in the formation of the recirculating bulge region in coastal buoyant outflows. J. Mar. Res., 61, 411-434.

2003b. The characteristics of the recirculating bulge region in coastal buoyant outflows. J. Mar. Res., 61, 435-463.

Boormans, M. and C. Garrett. 1989. A simple criterion for gyre formation by the surface outflow from a strait, with application to the Alboran Sea. J. Geophys. Res., 94, 12,637-12,644.

Crank, J. 1975. The Mathematics of Diffusion, 2nd edition, Oxford University Press, 414 pp.

Davis, J. T. and E. K. Rideal. 1963. Interfacial Phenomena, Academic Press, NY, 480 pp.

Fischer, H. B. 1978. On the tensor form of the bulk dispersion coefficient in a bounded skewed shear flow. J. Geophys. Res., 83, 2373-2375.

Fong, D. A. and W. R. Geyer. 2001. Response of a river plume during an upwelling favorable wind event. J. Geophys. Res., 106, 1067-1084.

Fratantoni, P. S. and M. S. McCartney. 2010. Freshwater export from the Labrador Current to the North Atlantic Current at the Tail of the Grand Banks of Newfoundland. Deep-Sea Res. I, 57, $258-283$.

Garrett, C. J. R. and J. W. Loder. 1981. Dynamical aspects of shallow sea fronts. Phil. Trans. Roy. Soc., London A, 302, 563-581.

Garvine, R. W. 1987. Estuary plumes and fronts in shelf waters: A layer model. J. Phys. Oceanogr., 17, 1877-1896.

1995. A dynamical system for classifying buoyant coastal discharges. Cont. Shelf Res., 15, $1585-1596$.

1999. Penetration of buoyant coastal discharge onto the continental shelf: A numerical model experiment. J. Phys. Oceanogr., 29, 1892-1909. 
Gill, A. E. 1981. Homogeneous intrusions in a rotating stratified fluid. J. Fluid Mech., 103, 275-295. 1982. Atmosphere-Ocean Dynamics, Volume 30 of Int. Geophys. Ser., Academic, San Diego, CA, $662 \mathrm{pp}$.

Gill, A. E., J. M. Smith, R. P. Cleaver, R. Hide, and P. R. Jonas. 1979. The vortex created by mass transfer between layers of a rotating fluid. Geophys. Astro. Fluid Dyn., 12, 195-220.

Gregorio, S. O., D. B. Haidvogel, P. J. Thomas, E. S. Taskinoglu, and A. J. Skeen. 2011. Laboratory and numerical simulations of gravity-driven coastal currents: Departures from geostrophic theory. Dyn. Atmos. Oceans, 52, 20-50.

Griffiths, R. W. 1986. Gravity currents in rotating systems. Ann. Rev. Fluid Mech., 18, 59-89.

Griffiths, R. W. and E. J. Hopfinger. 1983. Gravity currents moving along a lateral boundary in a rotating frame. J. Fluid Mech., 134, 357-399.

Griffiths, R. W. and P. F. Linden. 1981. The stability of buoyancy-driven coastal currents. Dyn. Atmos. Oceans, 5, 281-306.

Hacker, J. N. and P. F. Linden. 2002. Gravity currents in rotating channels. Part I. steady-state theory. J. Fluid Mech., 457, 295-324.

Hermann, A. J., P. B. Rhines, and E. R. Johnson. 1989. Nonlinear Rossby adjustment in a channel: Beyond Kelvin waves. J. Fluid Mech., 205, 469-502.

Hetland, R. D. 2005. Relating river plume structure to vertical mixing. J. Phys. Oceanogr., 35, 16671688.

Horner-Devine, A. R., D. E. Fong, S. G. Monismith, and T. Maxworthy. 2006. Laboratory experiments simulating a coastal river inflow. J. Fluid Mech., 555, 203-232.

Horner-Devine, A. R., D. A. Jay, P. M. Orton, and E. Y. Spahn. 2009. A conceptual model of the strongly tidal Columbia River plume. J. Marine Sys., 78, 460-475.

Klinger, B. A. 1994. Inviscid current separation from rounded capes. J. Phys. Oceanogr., 24, 18051811.

Landau, L. D. and E. M. Lifshitz. 1959. Fluid Mechanics. Course of Theoretical Physics, 6, Pergamon Press. 536 pp.

Lentz, S. 2008. Obervations and a model of the mean circulation over the Middle Atlantic Bight continental shelf. J. Phys. Oceanogr., 38, 1203-1221.

Lentz, S. and K. Helfrich. 2002. Buoyant gravity currents along a sloping bottom in a rotating fluid. J. Fluid Mech., 464, 251-278.

Levich, V. G. and V. S. Krylov. 1969. Surface-tension-driven phenomena. Ann. Rev. Fluid. Mech., 1, 293-316.

Loder, J., B. Petrie, and G. Gawarkiewicz. 1998. The coastal ocean off northeastern North America: A large-scale view in The Sea: The Global Coastal Ocean. Regional Studies and Syntheses, volume 11, John Wiley and Sons, 105-133.

MacCready, P. and P. B. Rhines. 1991. Buoyant inhibition of Ekman transport on a slope and its effect on stratified spin-up. J. Fluid Mech., 223, 631-661.

MacDonald, D. G. and W. R. Geyer. 2004. Turbulent energy production and entrainment at a highly stratified estuarine front. J. Geophys. Res., 109, C05004, doi:10.1029/2003JC002094.

McClimans, T. A. and S. Saegrov. 1982. River plume studies in distorted Froude models. J. Hydr. Res., 20, 15-27.

Münchow, A. and R. W. Garvine. 1993. Buoyancy and wind forcing of a coastal current. J. Mar. Res., 51, 293-322.

Neumann, G. and W. J. Pierson. 1966. Principles of Physical Oceanography, Prentice-Hall, Inc., NJ, $545 \mathrm{pp}$.

Nof, D. 1987. Penetrating outflows and the dam-breaking problem. J. Mar. Res., 45, 557-577.

Nof, D. and T. Pichevin. 2001. The ballooning of outflow. J. Phys. Oceanogr., 31, 3045-3058. 
Pattle, R. E. 1959. Diffusion from an instantaneous point source with a concentration-dependent coefficient. Quat. J. Mech. and Appl. Math., 12, 407-409.

Pedlosky, J. 1987. Geophysical Fluid Dynamics, 2nd ed., Springer-Verlag: NY, 710 pp.

Pimenta, F. M., A. D. Kirwan Jr., and P. Huq. 2011. On the transport of buoyant coastal plumes. J. Phys. Oceanogr., 41, 620-640.

Pritchard, M. and D. A. Huntley. 2006. A simplified energy and mixing budget for a small river plume discharge. J. Geophys. Res., 111, C03019, doi: 10.1019/2005JC002984.

Rossby, T. and J. Nilsson. 2003. Current switching as the cause of rapid warming at the end of the Last Glacial Maximum and Younger Dryas. Geophys. Res. Let., 30, doi:10.1029/2002GL015423.

Sanders, T. M. and R. W. Garvine. 2001. Fresh water delivery to the continental shelf and subsequent mixing: An observational study. J. Geophys. Res., 106, 27,087-27,101.

Smolarkiewicz, P. K. and T. L. Clark. 1986. The multidimensional positive definite advection transport algorithm: Further development and applications. J. Comput. Phys., 67, 396-438.

Smolarkiewicz, P. K. and L. G. Margolin. 1998. MPDATA: A finite-difference solver for geophysical flows. J. Comput. Phys., 140, 459-480.

Stern, M. E. 1980. Geostrophic fronts, bores, breaking and blocking waves. J. Fluid Mech., 99, 687703.

Stern, M. E., J. A. Whitehead, and B. L. Hua. 1982. The intrusion of the head of a gravity current along the coast of a rotating fluid. J. Fluid Mech., 123, 237-266.

Stommel, H. and K. N. Fedorov. 1967. Small scale structure in temperature and salinity near Timor and Mindanao. Tellus, 19, 306-325.

Thomas, P. J. and P. F. Linden. 2007. Rotating gravity currents: Small-scale and large-scale laboratory experiments and a geostrophic model. J. Fluid Mech., 578, 35-65.

Vallis, G. K. 2006. Atmospheric and Ocean Fluid Dynamics: Fundamentals and Large-Scale Circulation, Cambridge University Press. 745 pp.

Weast, R. C. and M. J. Astle. 1981. CRC handbook of Chemistry and Physics (62nd edition), CRC Press, Inc.

Whitehead, J. A. and A. R. Miller. 1979. Laboratory simulation of the gyre in the Alboran Sea. J. Geophys. Res., 84, 3733-3742.

Whitehead, J. A., A. Leetmaa, and R. A. Knox. 1974. Rotating hydraulics of strait and sill flows. Geophys. Fluid Dyn., 6, 101-125.

Wright, D. G. 1989. On the alongshelf evolution of an idealized density front. J. Phys. Oceanogr., 79, 532-541.

Yankovsky, A. E. and D. C. Chapman. 1997. A simple theory for the fate of buoyant coastal discharges. J. Phys. Oceanogr., 27, 1386-1401.

Received: 28 February, 2011; revised: 25 August, 2011. 